\title{
Molecular and Functional Characterization of Neurogenin-2 Induced Human Sensory Neurons
}

\section{OPEN ACCESS}

Edited by:

Daniela Pietrobon,

University of Padua, Italy

Reviewed by:

Bernard Fioretti,

University of Perugia, Italy

Francesco Ferrini,

University of Turin, Italy

*Correspondence:

Mirella Dottori

mdottori@uow.edu.au

Rocio K. Finol-Urdaneta

rfinolu@uow.edu.au

tThese authors have contributed equally to this work

Specialty section: This article was submitted to

Cellular Neurophysiology,

a section of the journal

Frontiers in Cellular Neuroscience

Received: 31 August 2020 Accepted: 13 November 2020 Published: 04 December 2020

Citation:

Hulme AJ, McArthur JR, Maksour S, Miellet S, Ooi L, Adams DJ, Finol-Urdaneta RK and Dottori M (2020) Molecular and Functional Characterization of Neurogenin-2 Induced Human Sensory Neurons. Front. Cell. Neurosci. 14:600895. doi: 10.3389/fncel.2020.600895

\begin{abstract}
Amy J. Hulme ${ }^{1,2+}$, Jeffrey R. McArthur ${ }^{1,2 t}$, Simon Maksour ${ }^{1,2}$, Sara Miellet ${ }^{1,2}$, Lezanne Ooi ${ }^{1,3,4}$, David J. Adams ${ }^{1,2,4}$, Rocio K. Finol-Urdaneta ${ }^{1,2 *}$ and Mirella Dottori ${ }^{1,2,4 *}$

IIllawarra Health and Medical Research Institute, Wollongong, NSW, Australia, ${ }^{2}$ School of Medicine, University of Wollongong, Wollongong, NSW, Australia, ${ }^{3}$ School of Chemistry and Molecular Bioscience, University of Wollongong, Wollongong, NSW, Australia, ${ }^{4}$ Molecular Horizons, University of Wollongong, Wollongong, NSW, Australia
\end{abstract}

Sensory perception is fundamental to everyday life, yet understanding of human sensory physiology at the molecular level is hindered due to constraints on tissue availability. Emerging strategies to study and characterize peripheral neuropathies in vitro involve the use of human pluripotent stem cells (hPSCs) differentiated into dorsal root ganglion (DRG) sensory neurons. However, neuronal functionality and maturity are limited and underexplored. A recent and promising approach for directing hPSC differentiation towards functionally mature neurons involves the exogenous expression of Neurogenin-2 (NGN2). The optimized protocol described here generates sensory neurons from hPSC-derived neural crest (NC) progenitors through virally induced NGN2 expression. NC cells were derived from hPSCs via a small molecule inhibitor approach and enriched for migrating NC cells (66\% SOX10+ cells). At the protein and transcript level, the resulting NGN2 induced sensory neurons ( $\mathrm{NGN}_{2} \mathrm{iSNs}$ ) express sensory neuron markers such as BRN3A (82\% BRN3A+ cells), ISLET1 (91\% ISLET1+ cells), TRKA, TRKB, and TRKC. Importantly, NGN2iSNs repetitively fire action potentials (APS) supported by voltage-gated sodium, potassium, and calcium conductances. In-depth analysis of the molecular basis of ${ }_{N G N 2}$ iSN excitability revealed functional expression of ion channels associated with the excitability of primary afferent neurons, such as Nav1.7, Nav1.8, Kv1.2, Kv2.1, BK, Cav2.1, Cav2.2, Cav3.2, ASICs and HCN among other ion channels, for which we provide functional and transcriptional evidence. Our characterization of stem cell-derived sensory neurons sheds light on the molecular basis of human sensory physiology and highlights the suitability of using hPSC-derived sensory neurons for modeling human DRG development and their potential in the study of human peripheral neuropathies and drug therapies.

Keywords: dorsal root ganglia, electrophysiology, excitability, human sensory neurons, ion channels, NGN2, pluripotent stem cells 


\section{INTRODUCTION}

Basic sensory experiences such as pain, temperature, pressure, and spatial positioning require specialized sensory neurons within the peripheral nervous system to detect and transmit stimuli to the central nervous system for processing. Different subtypes of sensory neurons have specific functions in detecting and conveying signals of proprioception (detection of spatial position, movement, muscle pressure, and tension); mechanoreception (cutaneous touch, hair deflection, and vibration), and nociception (pain perception, noxious thermal, mechanical and chemical stimuli). As can be expected with multiple sensory subtypes, there is a plethora of diseases and sensory neuropathies associated with dysfunction in the development or function of each neuronal subtype within the dorsal root ganglia (DRG). These can be caused by, but are not limited to, acquired or inherited/genetic diseases, autoimmune disorders, inflammation, injury, and idiopathic conditions that can result in hypersensitivity, numbness, ataxia, and chronic pain (Johnson et al., 1986; Kuntzer et al., 2004; Sghirlanzoni et al., 2005; Axelrod and Gold-Von Simson, 2007; Damasceno et al., 2008; Martinez et al., 2012). Furthermore, adverse side effects of treatments (e.g., chemotherapeutics for the treatment of cancer) can lead to damage of sensory neurons with peripheral neuropathic symptoms like numbness, chronic pain, and/or tingling. At the cellular level, dysfunction, and damage to sensory neurons can alter the expression, distribution, density, and conductance of membrane ion channels. These changes can dramatically alter action potential (AP) transduction producing changes in the excitability of specific sensory neurons resulting in the symptoms of peripheral neuropathies (Rasband et al., 2001; Chaplan et al., 2003; Chen et al., 2009; Zhang and Dougherty, 2014; Pitake et al., 2019). For example, injury to rodent paws causes an increase in the expression of specific calcium channels in the membrane of sensory neurons resulting in hyper-excitable neurons (Chen et al., 2009). Similarly, chemotherapeutics can alter the expression of ion channels resulting in peripheral neuropathies (Descoeur et al., 2011; Zhang and Dougherty, 2014; Leo et al., 2017). Following application of paclitaxel, a common chemotherapeutic used for several types of cancers, rat DRG neurons manifest altered excitability due to higher expression of voltage-gated potassium, hyperpolarizationactivated cyclic nucleotide-gated $(\mathrm{HCN})$, and voltage-gated sodium channels together with a decrease in the expression of inwardly-rectifying potassium channels (Zhang and Dougherty, 2014). Ion channels have a critical role in the development and progression of peripheral neuropathies hence, strides have been made towards the development of cancer therapies aimed at curtailing excitability remodeling and neurotoxicity (Keenan et al., 2020).

Methods to investigate neuronal excitability, ion channel function and dysfunction in sensory neurons have previously relied on animal models. However, inherent species differences limit the translation of rodent-based findings to human neurophysiology and pathology (Zylka et al., 2003; Han et al., 2015; Chang et al., 2018; Rostock et al., 2018; Schwaid et al., 2018). To circumvent the translatability of rodent models and the difficulty in accessing human tissue, the use of human pluripotent stem cell (hPSC) technology has rapidly progressed. These approaches enable human neurons to be derived in vitro to investigate all aspects of neuronal function including development, toxicity and diseases while providing a relevant platform for drug screening and delivery.

There are various protocols to derive DRG sensory neurons from hPSCs (Chambers et al., 2012; Blanchard et al., 2015; Boisvert et al., 2015; Schrenk-Siemens et al., 2015; Wainger et al., 2015; Alshawaf et al., 2018; Nickolls et al., 2020). One approach uses small molecule inhibitors to generate a progenitor cell, such as neural crest (NC) cells, which are then transduced to express the transcription factors of interest to drive the differentiation of the NC cells to functional sensory neurons (Schrenk-Siemens et al., 2015; Nickolls et al., 2020). The benefit of this approach is that it mimics neurogenesis and developmental patterning by causing the expression of transcription factors at specific developmental time points. Studies following this method report improved sensory neuron efficiency compared to those that bypass the progenitor states (Schrenk-Siemens et al., 2015; Nickolls et al., 2020). For example, Schrenk-Siemens et al. (2015) and Nickolls et al. (2020) have successfully used small molecules to generate NC cells in which either the expression of Neurogenin-2 (NGN2) or a combination of NGN2 and BRN3A is introduced at the $\mathrm{NC}$ stage to mimic the first wave of neurogenesis producing functional sensory neurons (Schrenk-Siemens et al., 2015; Nickolls et al., 2020). Transcriptional analysis of sensory neuron cultures generated with the available protocols has evidenced good efficiency, however, the resulting sensory neuron excitability profiles and functional expression of the underlying conductances have not been thoroughly addressed. Functional characterization of the resulting neuroexcitability profiles is of particular importance, as emerging evidence suggests that the pathophysiology of many sensory neuropathies results from the dysfunction of their electrophysiological properties often arising from a channelopathy. Furthermore, a thorough understanding of the voltage-gated sodium, calcium, and potassium currents underpinning excitability sets the foundation to establish hPSC-derived sensory neuron cultures as platforms for drug screening, drug delivery, and disease modeling. In this study, sensory neurons were derived from hPSCs using a combination of small molecules to generate NC progenitors followed by induced expression of NGN2. This work provides a detailed description of human NGN2 derived sensory neuron's $\left(\mathrm{NGN}_{2} \mathrm{iSN}\right)$ major voltage-gated sodium, calcium and potassium conductances, as well as their functional acid-sensitive currents. The observed excitability profile of ${ }_{\mathrm{NGN}_{2}} \mathrm{iSNs}$ demonstrates the generation of a functionally heterogeneous population of sensory neurons with membrane passive and active electrical properties akin to mature human sensory neurons.

\section{MATERIALS AND METHODS}

\section{hPSC Culture}

All experiments were approved by the University of Wollongong Human Ethics Committee (\#2017-375) and University of 
Wollongong Institutional Biosafety Committee (\#GT19-09). The H9 human stem cell line (WA09, WiCell) was maintained on vitronectin $\mathrm{XF}^{\mathrm{TM}} \quad$ (\#07180, StemCell Technologies) coated T25 flasks using TeSR-E8 (\#5990, STEMCELL Technologies), at $37^{\circ} \mathrm{C} 5 \% \mathrm{CO}_{2}$. When the cultures reached a confluence of $60-70 \%$, they were passaged using $0.5 \mathrm{mM}$ EDTA/PBS ${ }^{-1-}$.

\section{Lentiviral Production}

HEK293T cells were maintained in DMEM/F12 5\% Foetal Bovine Serum (FBS; SFBS-F, Interpath) in a T75 flask, passaged using accutase (00-4555-56, Life Technologies), and seeded at a density of $4 \times 10^{6}$ cells/flask, $24 \mathrm{~h}$ before transfection. Lentiviral particles were produced in HEK293T cells through co-transfection of the doxycycline-inducible lentiviral vector PLV-TetO-hNGN2-eGFP-PURO (\#79823, Addgene) or the reverse tetracycline transactivator vector FUW-M2rtTA (\#20342, Addgene) or PLV-TetO-eGFP-PURO (modification of \#79823 Addgene vector) with the packaging plasmids vSVG (\#8454, Addgene), RSV (\#12253, Addgene) and pMDL (\#12251, Addgene) using polyethyleneimine (408727, Sigma-Aldrich) and Opti-MEM (\#31985062, Life Technologies). The media from the transfected HEK293T cells was changed $6 \mathrm{~h}$ after transfection and replaced with DMEM/F12 5\% FBS. Viral particles were collected at 24,48 , and $72 \mathrm{~h}$ post-transfection, filtered $(0.45 \mu \mathrm{m}$ pore size), and centrifuged at $66,000 \times$ $g$ for $2.5 \mathrm{~h}$ at $4^{\circ} \mathrm{C}$. The pelleted viral particles were resuspended in PBS at a $200 \times$ enrichment, aliquoted, and stored at $-80^{\circ} \mathrm{C}$ until use. The viral particles were tested on hPSCs to confirm successful production (Supplementary Figure 1).

\section{hPSC Differentiation to Sensory Neurons}

hPSC differentiation to sensory neurons was based on previously published methods by Alshawaf et al. (2018), with modifications. Briefly, hPSC was seeded in an organ culture dish $(60 \times 15 \mathrm{~mm}$, \#353037, Corning) previously coated with $10 \mu \mathrm{g} / \mathrm{ml}$ laminin (\#23017015, Thermo Fisher Scientific) for $24 \mathrm{~h}$ at $4^{\circ} \mathrm{C}$, at a density of $2 \times 10^{4}$ cells/dish in TeSR-E8. Following $24 \mathrm{~h}$ (day 1), the media was removed and replaced with neural induction media [Neurobasal medium (\#21103-049, Thermo Fisher Scientific), DMEM/F12, $1 \times$ N-2 supplement (\#17502-048, Thermo Fisher Scientific), 1× B-27 supplement (without vitamin A; \#12587-010, Thermo Fisher Scientific), $1 \times$ Insulin-transferrin-Selenium-A (51300-044, Thermo Fisher Scientific), 2 mM L-glutamine (25030149, Life Technologies), $0.3 \%$ glucose (G8769, Sigma-Aldrich)], supplemented with $3 \mu \mathrm{M}$ CHIR99021 (SML1046, Sigma-Aldrich) and $10 \mu \mathrm{M}$ SB431524 (72234, STEMCELL Technologies) and this was repeated on day 3 to direct the differentiation to caudal neural progenitors (CNPs). Neurospheres were formed on day 5 by harvesting the CNPs using $0.5 \mathrm{mM} \mathrm{EDTA/PBS}{ }^{-/-}$, centrifuged at $200 \times g$ for $3 \mathrm{~min}$. CNPs were then resuspended in neuronal media [Neurobasal medium (\#21103-049, Thermo Fisher Scientific), $1 \times \mathrm{N}-2$ supplement (\#17502-048, Thermo Fisher Scientific), $1 \times$ B-27 supplement (without vitamin A; \#12587-010, Thermo Fisher Scientific), $1 \times$ Insulin-transferrin-
Selenium-A (\#51300-044, Thermo Fisher Scientific), $2 \mathrm{mM}$ L-glutamine (\#25030149, Life Technologies)] supplemented with $20 \mathrm{ng} / \mathrm{ml} \mathrm{FGF2} \mathrm{( \# 78003,} \mathrm{STEMCELL} \mathrm{Technologies)} \mathrm{and}$ $10 \mathrm{ng} / \mathrm{ml}$ BMP2 (RDS355BM010, in vitro Technologies) and $100 \mu \mathrm{l}$ of cell suspension plated into each well of an ultra-low attachment U-bottom 96 well plate (CLS7007, Sigma-Aldrich) and centrifuged at $200 \times g$ for $4 \mathrm{~min}$. Neurosphere formation was observed following $24 \mathrm{~h}$, and $50 \mu \mathrm{l} /$ well of neuronal media supplemented with $20 \mathrm{ng} / \mathrm{ml} \mathrm{FGF2}$ and $10 \mathrm{ng} / \mathrm{ml} \mathrm{BMP2}$ was added on day 8 , with full media changes conducted every 3rd day. Before plating the spheres, $12 \mathrm{~mm}$ glass coverslips in 24-well plates were coated in $10 \mu \mathrm{g} / \mathrm{ml}$ Poly-D-Lysine (P6407, Sigma-Aldrich) for $30 \mathrm{~min}$ at room temperature and then $10 \mu \mathrm{g} / \mathrm{ml}$ laminin at $4^{\circ} \mathrm{C}$ overnight. To enrich for the migrating $\mathrm{NC}$, on day 12 the neurospheres were plated as whole spheres on the previously coated coverslips in neuronal media supplemented with $10 \mu \mathrm{M}$ Y-27632 (72302, STEMCELL Technologies). On day 14, the neurospheres were removed using a P200 pipette, leaving behind the migrating NC cells. These cells were then transduced with $2 \mu \mathrm{l} / \mathrm{ml}$ PLV-TetOhNGN2-eGFP-PURO or PLV-TetO-eGFP-PURO lentiviral particles and $2 \mu \mathrm{l} / \mathrm{ml}$ FUW-M2rtTA lentiviral particles in neuronal media supplemented with $10 \mu \mathrm{M}$ Y-27632, $10 \mathrm{ng} / \mathrm{ml}$ BDNF (78005, STEMCELL Technologies), $10 \mathrm{ng} / \mathrm{ml}$ GDNF (78058, STEMCELL Technologies), 10 ng/ml NT-3 (78074, STEMCELL Technologies) and $10 \mathrm{ng} / \mathrm{ml}$ ß-NGF (78092, STEMCELL Technologies). To induce NGN2 expression a full media change was conducted on day 15 removing the viral medium and replacing it with neuronal media supplemented with $1 \mu \mathrm{g} / \mathrm{ml}$ doxycycline (D9891, Sigma-Aldrich), $10 \mu \mathrm{M}$ Y-27632, 10 ng/ml BDNF, $10 \mathrm{ng} / \mathrm{ml}$ GDNF, 10 ng/ml NT-3 and $10 \mathrm{ng} / \mathrm{ml} B$-NGF. NGN2 expression was induced by the addition of doxycycline for $96 \mathrm{~h}$ (day 15-19). To select for successfully transduced cells $0.5 \mu \mathrm{g} / \mathrm{ml}$ of puromycin (73342, STEMCELL Technologies) was added for $48 \mathrm{~h}$ (day 17-19). Media changes were conducted every 2-3 days. On day 22, BrainPhys ${ }^{\mathrm{TM}}$ Neuronal Medium (05790, STEMCELL Technologies), $0.02 \%$ NeuroCult ${ }^{\mathrm{TM}}$ SM1 Without Vitamin A (05731, STEMCELL Technologies), 0.01\% N2 Supplement-A (07152, STEMCELL Technologies) was phased into the neuronal media to mature the neurons $(25: 75,50: 50,75: 25$, 100:0 Brainphys: neuronal media every media change) and proliferating cells were removed upon addition of an anti-mitotic agent $2.5 \mu \mathrm{M}$ cytosine $\beta$-D-arabinofuranoside (AraC; C1768, Sigma-Aldrich) between days 25-27 for $48 \mathrm{~h}$. The neurons were matured until day 34 and were then fixed for immunocytochemistry or harvested for RNA. For patch-clamp analysis, the neurons were used between days 34-48.

\section{Immunocytochemistry}

Cells were washed with PBS three times and fixed with $4 \%$ PFA for $20 \mathrm{~min}$ at room temperature and then washed three times with PBS. Cells were permeabilized with $0.1 \%$ triton/PBS for $10 \mathrm{~min}$ and then blocked in $10 \%$ donkey serum/PBS (D9663, Sigma-Aldrich) for $1 \mathrm{~h}$ at room temperature. Samples were then incubated with the primary antibody in 
TABLE 1 | Primary and secondary antibody details and dilutions.

\begin{tabular}{lll}
\hline Antibody name & The catalog number, company & Dilution \\
\hline Mouse anti-P75NTR & M-1818-100, Biosensis & $1: 500$ \\
Goat anti-SOX10 & RDSAF2864, R\&D Systems & $1: 100$ \\
Mouse anti-PAX3/7 & Sc-365843, Santa Cruz & $1: 500$ \\
& Biotechnology & \\
Rabbit anti-ISLET1 & ab20670, Abcam & $1: 500$ \\
Mouse anti-BRN3A & MAB1585, Millipore & $1: 500$ \\
Mouse anti-PERIPHERIN & MAB1527, Millipore & $1: 500$ \\
Mouse anti-B- III TUBULIN & MAB1637, Millipore & $1: 500$ \\
Goat anti-TRKA & RDSAF175, R\&D Systems & $1: 400$ \\
Mouse anti-TRKB & NOVNBP147898, Novus & $1: 100$ \\
Rabbit anti-TRKC & Biologicals & \\
Rabbit anti-Nav1.8 & 7H3L20, Thermo Fisher Scientific & $1: 250$ \\
Rabbit anti-Nav1.7 & ab66743, Abcam & $1: 200$ \\
Guinea pig anti-ASIC3 & ab65167, Abcam & $1: 500$ \\
Rabbit anti-PAN-ASIC & AGP-052, Alomone & $1: 500$ \\
Rabbit anti-TRPV1 & ASIC-PAN-51A, Alpha & $1: 100$ \\
Donkey-anti-mouse IgG-488 & Diagnostic & \\
Donkey-anti-rabbit IgG-555 & NB120-3487, Novus Biologicals & $1: 500$ \\
Donkey-anti-goat IgG-647 & ab150062, Abcam & $1: 500$ \\
Donkey-anti-mouse IgG-647 & ab150135, Abcam & $1: 500$ \\
Goat Anti-Guinea pig IgG & ab150186, Abcam & $1: 500$ \\
H\&L-555 & & $1: 500$ \\
& & $1: 500$ \\
& &
\end{tabular}

$10 \%$ donkey serum/PBS overnight at $4^{\circ} \mathrm{C}$. The primary and secondary antibodies were used as per Table 1. Following the overnight incubation, the coverslips were washed with PBS three times for $5 \mathrm{~min}$ and then incubated with the appropriate secondary antibody in $10 \%$ donkey serum/PBS for $1 \mathrm{~h}$ at room temperature. Samples were again washed three times for $5 \mathrm{~min}$ in PBS and stained with DAPI (D9542, Sigma-Aldrich) for $15 \mathrm{~min}$ and then washed three times for $5 \mathrm{~min}$ in PBS. The coverslips were mounted with ProLong ${ }^{\text {TM }}$ Gold Antifade Mountant (P36934, Life Technologies Australia) onto microscope slides (MENSF41296P, Thermo Fisher Scientific). Images were taken using a Leica confocal SP8 microscope and were exported and analyzed using ImageJ software.

\section{RNA Purification, cDNA, and RT-qPCR}

RNA was isolated and purified using the PureLink ${ }^{\mathrm{TM}}$ RNA Mini Kit (12183025, Thermo Fisher Scientific) kit according to the manufacturer's instructions. Genomic DNA was removed, and RNA was reverse transcribed into cDNA using the iScript ${ }^{\mathrm{TM}}$ gDNA Clear cDNA Synthesis Kit (Biorad, 1725035) in a master cycler. cDNA was diluted to $20 \mathrm{ng} / \mu \mathrm{l}$ and stored at $-20^{\circ} \mathrm{C}$ until used for RT-qPCR. RT-qPCR was conducted using the PowerUP SYBR green master mix (A25778, Thermo Fisher Scientific) and respective primers (Table 2) in a Quantistudio 5, following the manufacturer's instructions. All targets were internally normalized to three housekeeper genes, GAPDH, $P P I A$, and $B 2 M$, and expressed as $2^{-\Delta \mathrm{Ct}}$.

\section{Electrophysiology}

Whole-cell patch-clamp recordings of $\mathrm{NGN}_{2} \mathrm{iSNs}$ were made at room temperature $\left(20-22^{\circ} \mathrm{C}\right)$ with a MultiClamp $700 \mathrm{~B}$ Amplifier, digitalized with a Digidata 1440 and controlled with pClamp11 software (Molecular Devices, San Jose, CA, USA). Whole-cell membrane currents were sampled at $100 \mathrm{kHz}$, filtered at $10 \mathrm{kHz}$, and series resistance compensated 60-80\%. Fire-polished borosilicate (1B150F-4, World Precision Instruments, USA) patch pipettes were used with resistance 2-4 $\mathrm{M} \Omega$ and filled with intracellular solutions containing (in $\mathrm{mM}$ ): $140 \mathrm{~K}$-Gluconate, $10 \mathrm{NaCl}, 2 \mathrm{MgCl}_{2}, 5 \mathrm{EGTA}$, and 10 HEPES, $\mathrm{pH}$ 7.2. The resting membrane potential (RMP) was recorded immediately after switching into the current-clamp mode as the average membrane voltage in the absence of current injection. Stimulus membrane potentials were determined by injection of increasing bias currents. The rheobase or current threshold was defined as the minimum amount of current necessary to evoke a single AP during $500 \mathrm{~ms}$ depolarizing current steps (in $5 \mathrm{pA}$ increments). Elicited APs were counted/plotted as a function of the current injection intensity during stimulation. For voltage-clamp experiments, the extracellular solution was varied depending on the ion channel being examined. For voltage-gated sodium channels, $\mathrm{Na}^{+}$currents $\left(I_{\mathrm{Na}}\right)$ were isolated with an extracellular solution containing (in mM): $110 \mathrm{NaCl}, 2 \mathrm{CaCl}_{2}, 2 \mathrm{MgCl}_{2}, 30 \mathrm{TEA}-\mathrm{Cl}$, 10 D-Glucose, and 10 HEPES, $\mathrm{pH}$ 7.3. To isolate $\mathrm{Ca}^{2+}$ currents $\left(I_{C a}\right)$, the extracellular solution contained (in $\mathrm{mM}$ ): $140 \mathrm{TEA}-\mathrm{Cl}$, $10 \mathrm{mM} \mathrm{CaCl}_{2}, 1 \mathrm{MgCl}_{2}, 10$ HEPES, 10 D-Glucose, pH 7.3. For current-clamp experiments an extracellular solution containing (in $\mathrm{mM}$ ): $135 \mathrm{NaCl}, 2 \mathrm{CaCl}_{2}, 2 \mathrm{MgCl}_{2}, 5 \mathrm{KCl}, 10 \mathrm{D}$-Glucose, 10 HEPES, pH 7.3 was used. To examine $K^{+}$currents $\left(I_{\mathrm{K}}\right)$ the latter extracellular solution was supplemented with $1 \mu \mathrm{M}$ TTX. Voltage dependence of activation and steady-state inactivation (SSI) of the various ionic currents were fit by the modified Boltzmann equation:

$$
I \text { or } G=1 /\left(1+\exp \left(\frac{V m-V_{0.5}}{k a}\right)\right)
$$

where $I$ is the current or $G$ is the conductance, $V m$ is the pre-pulse potential, $V_{0.5}$ is the half-maximal activation potential and $k a$ is the slope factor.

\section{Pharmacology}

Tetrodotoxin citrate was purchased from Abcam (Melbourne, Australia). Kv channel inhibitors were sequentially and cumulatively applied using the most selective antagonists (at concentrations where target selectivity has been verified experimentally) first followed by less selective/potent modulators. Cone snail toxin $\kappa$-RIIIJ (B. Olivera, University of Utah) selectively blocks heteromeric Kv1.2 complexes with Kv1.1 or Kv1.6 channels (Cordeiro et al., 2019). Scorpion toxin, Urotoxin (Uro, Alomone Labs, Cat\#: STU-200), is a potent inhibitor of homomeric Kv1.2 (Luna-Ramirez et al., 2020). Guangxitoxin-1E (GxTx, Alomone Labs, Cat\#: STG-200) was applied (Liu and Bean, 2014) to inhibit Kv2 channels. Subsequent application of 4-aminopyridine (4-AP, Sigma-Aldrich, A78403) was applied to block all Kv3 channels (Gutman et al., 2005) and was followed by AmmTx3 (Smartox Biotechnology, Saint-Egrève, France, AMX001-50010) to inhibit Kv4 channels (Vacher et al., 2002). 
TABLE 2 | Primer sequences.

\begin{tabular}{|c|c|c|c|}
\hline Gene name & Protein name & FW primer $5^{\prime}-3^{\prime}$ & REV primer $5^{\prime}-3^{\prime}$ \\
\hline GAPDH & GAPDH & TCGGAGTCAACGGATTTGGT & TTCCCGTTCTCAGCCTTGAC \\
\hline PPIA & PPIA & ACGTGGTATAAAAGGGGCGG & CTGCAAACAGCTCAAAGGAGAC \\
\hline B2M & $\beta 2$ microglobulin & AAGGACTGGTCTTTCTATCTC & GATCCCACTTAACTATCTTGG \\
\hline NTRK2 & TRKB & TTATGTGGATCAAGACTCTCC & AAACCACAATTGGGTATCTG \\
\hline NTRK3 & TRKC & GATTATTACAGGGTGGGAGG & AAGCTCCATACATCACTCTC \\
\hline POU4F1 & BRN3A & AACTGGACCTCAAAAAGAAC & GATAACGGACACTCCAAATC \\
\hline SCN9A & Nav1.7 & AAAGGGAAAACAATCTTCCG & TGTACTCGACATIITTGGTC \\
\hline SCN10A & Nav1.8 & AAAGGAGAAGAAGTTCCAGG & GGTTAAAGGTGATCCATTGTG \\
\hline KCNA2 & $\mathrm{Kv} 1.2$ & AGCCCAGCCCAATCCTAGAG & CTTGGCTGACCAGAGACGTG \\
\hline CACNA1H & Cav3.2 & TCTGGGCTACATCCGGAACC & TCTCCCAGACGCTGATGACC \\
\hline CACNA1B & Cav2.2 & TTGCTTACAAGCGCCTGGTT & CAGCGTGGACGTGAAGTGAA \\
\hline CACNA1A & Cav2.1 & CCTACCGACATGCCCAACAG & АTCTCTGCCCATCTCTCGCA \\
\hline HCN4 & $\mathrm{HCN} 4$ & GACTTCAGATITTACTGGGAC & CATCCTTGAAGAAGGTGATG \\
\hline
\end{tabular}

\section{RESULTS}

\section{Induction of NGN2 Expression in hPSC-Derived Neural Crest Cells Generates Sensory Neurons (NGN2 $\mathrm{iSN}$ )}

We modified the previously established protocol (Alshawaf et al., 2018) that uses a combination of small molecules and growth factors to generate a mixed population of sensory neurons from hPSCs (Figure 1A). Briefly, hPSC were first differentiated to caudal neural progenitor cells using small molecule inhibitors of the GSK3 $\beta$ (CHIR99021) and activin/nodal pathways (SB431542). Caudal neural progenitors were further differentiated to NC by treatment with BMP2 (Denham et al., 2015; Alshawaf et al., 2018; Abu-Bonsrah et al., 2019). To mimic the stages of sensory neurogenesis, this study set out to transiently induce NGN2 expression in migrating NC cells, defined by the expression of SOX10 and P75 ${ }^{\text {NTR }}$. To enrich for the migrating NC population, neurospheres were plated onto a monolayer for $24 \mathrm{~h}$ or $48 \mathrm{~h}$ and compared to neurospheres that were disaggregated into single cells supplemented with Y-27632 (Figures 1B,C). Whole neurospheres plated for $24 \mathrm{~h}$ had a 5.9 -fold increase in SOX10 expression ( $84 \pm 6 \%$ SOX10+ cells) compared to disaggregated neurospheres $\left(14 \pm 1 \%\right.$ SOX $10+$ cells; $t_{(4)}=11.47$, $p=0.003$, Figure 1C). Similarly, whole neurospheres plated for $48 \mathrm{~h}$ had a 3.5 -fold increase SOX10 expression (66 $\pm 6 \%$ SOX10+ cells) compared to disaggregated neurosphere cultures $\left(19 \pm 3 \%\right.$ SOX10+ cells; $t_{(4)}=7.54, p=0.0017$, Figure 1C). Furthermore, the $48 \mathrm{~h}$ cultures of whole sphere migrating cells also co-expressed the $\mathrm{NC}$ marker $\mathrm{P} 75^{\mathrm{NTR}}$. Additionally, PAX3/7 expression was detected in all conditions (Supplementary Figure 2). Thus, $48 \mathrm{~h}$ of migration was considered sufficient for the enrichment of SOX10+/P75 $5^{\mathrm{NTR}}+$ NC cells.
As outlined in Figure 1, NGN2 expression was induced in enriched migrating NC cells. Cultures were assessed by immunocytochemistry and RT-qPCR analyses at day 34 to determine the expression of sensory DRG neuronal markers. Expression of the neuronal marker B-III-TUBULIN was evident throughout the cultures. Additionally, $\mathrm{NGN}_{2} \mathrm{iSN}$ expressed pan-sensory neuron markers, with $82 \%$ of neurons expressing BRN3A and 91\% expressing ISLET1 (Figures 2A,B). Furthermore, BRN3A, ISLET1 (encoded for by POU4F1 and ISL1, respectively), and $P R P H$ mRNA transcript levels were increased in the $\mathrm{NGN}_{2} \mathrm{iSN}$ cultures relative to cultures transduced with a GFP control vector (Figure 2C). Additionally, neurons were positive for the nociceptor marker TRKA, the mechanoreceptor marker TRKB, and the proprioceptor marker TRKC (encoded for by NTRK1, NTRK2, NTRK3, respectively, Figures 2A,D). These data suggest that induced expression of NGN2 in NC progenitors resulted in their differentiation to heterogeneous populations of DRG sensory neurons and therefore are referred to as "NGN2-induced sensory neurons" $\left(\mathrm{NGN}_{2} \mathrm{iSN}\right)$.

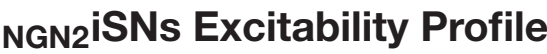

The passive and active electrical properties of the ${ }_{\mathrm{NGN}_{2}} \mathrm{iSN}$ were characterized by current clamp electrophysiological recordings in the whole-cell patch-clamp recording configuration. Standard neuronal excitability parameters, including resting membrane potential (RMP, $\mathrm{mV}$ ), rheobase ( $\mathrm{pA})$, number of APs at $2 \times$ rheobase (\#APs@ $2 \times$ rheobase), AP width (AP width@ 0 mV, $\mathrm{ms})$, and firing rate $(\mathrm{Hz})$ were evaluated upon the establishment of the whole-cell configuration and in response to small current injections. Representative current-clamp recordings of $\mathrm{NGN}_{2} \mathrm{iSNs}$ are depicted in Figure 3A displaying robust tonic neuronal firing. These neurons had an average RMP of $-56.4 \pm 0.9 \mathrm{mV}(n=21$, Figure 3B $)$ and cell capacitance of $12.7 \pm 1 \mathrm{pF}(n=21)$. Rheobase 


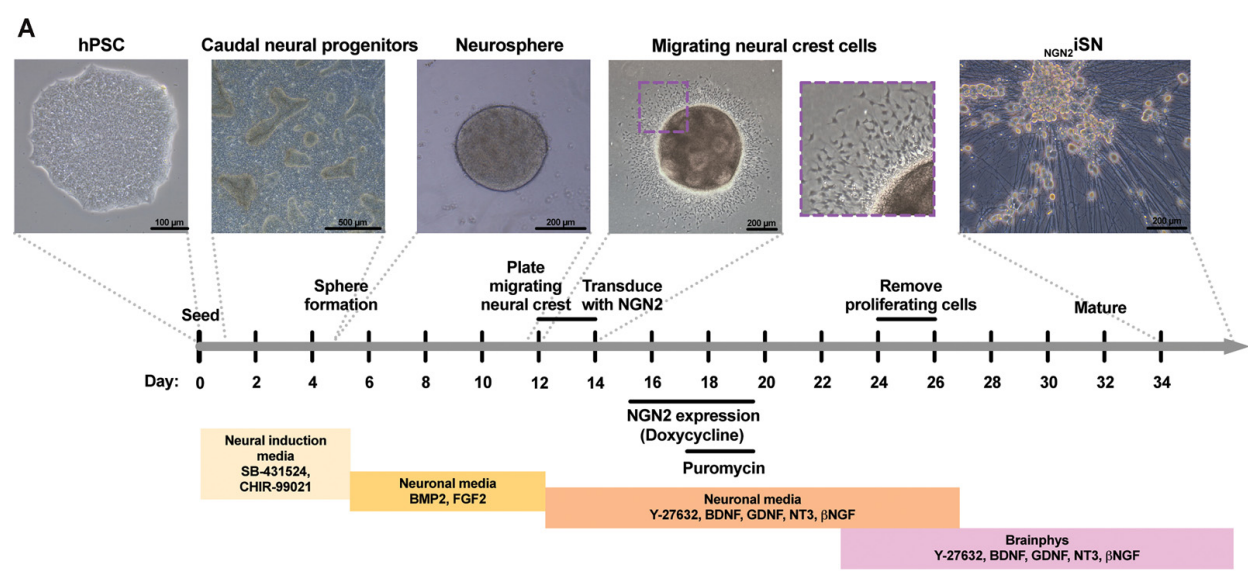

B

Whole

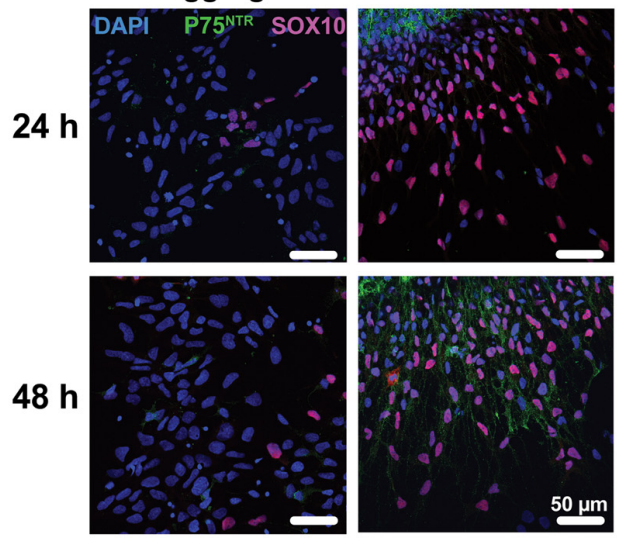

C

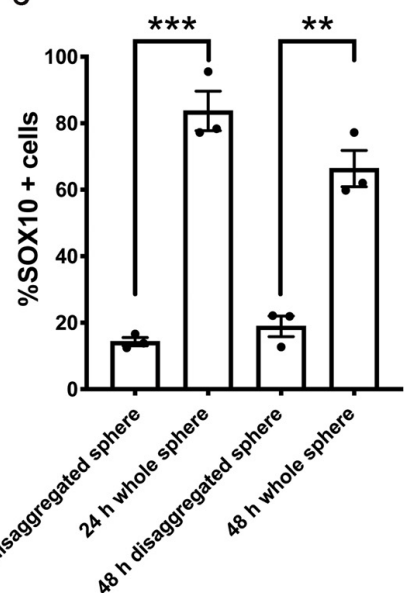

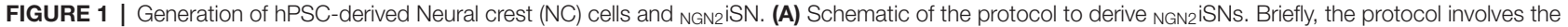
formation of caudal neural progenitors (days 1-5) and neurospheres (days 5-12). Neurospheres are plated down onto a monolayer (day 12) and then removed after $48 \mathrm{~h}$ (day 14), to enrich for NC cells. NC cells are transduced with the NGN2 and reverse tetracycline transactivator lentiviruses (day 14) and NGN2 expression is induced for $96 \mathrm{~h}$ (days 15-19) and successfully transduced cells are obtained via puromycin selection (days 17-19). Finally, proliferating cells are removed using an

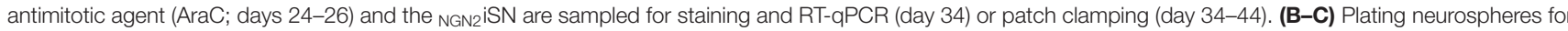
$48 \mathrm{~h}$ using the above protocol enriches for SOX10+ and P75NTR + NC cells. Neurospheres were either disaggregated into single cells or plated as whole spheres in NM supplemented with $10 \mu \mathrm{M}$ Y-27632 for $24 \mathrm{~h}$ or $48 \mathrm{~h}$. (B) Representative immunocytochemistry images of the NC markers P75 NTR (green) and SOX10 (pink). (C) The percentage of SOX10 positive cells, $n=3$ biological replicates, $>300$ cells counted per biological replicate, error bars presented as SEM, ${ }^{* *} p<0.01$, ${ }^{\star \star *} p<0.001$.

is defined as the current injection necessary to elicit a single AP and provides a measure of neuronal excitability. Firing of a single AP by the $\mathrm{NGN}_{2} \mathrm{iSN}$ shown in Figure 3A is highlighted in red (and presented as single trace "SN9" in Supplementary Figure 3 together with all other neurons included in the analysis). The average rheobase determined in these neurons was $15.5 \pm 2.0 \mathrm{pA}(n=21$, Figure $3 \mathrm{C})$. Accordingly, injection of a current equivalent to twice rheobase (shown in orange in Figure 3A) elicited on average $3.9 \pm 0.3 \mathrm{APs}(n=21$, Figure 3D). The mean AP width measured at $0 \mathrm{mV}$ was $3.0 \pm 0.2 \mathrm{~m}(n=$ 21, Figure 3E). A maximal firing rate of $13.1 \pm 1.0 \mathrm{~Hz}(n=21$, Figure 3F) was observed in this neuronal population. The first derivative $(\mathrm{d} V / \mathrm{d} t)$ of the APs fired at rheobase for all cells included in this study (presented in Supplementary Figure 3) revealed that $\sim 30 \%$ ( 6 out of 21 ) ${ }_{\mathrm{NGN}} \mathrm{iSN}$ displayed clearly defined humps arising from the inflection on the descending slope of the AP, which is typically associated with functional aspects of C-fiber nociceptive neurons in rodents (Ritter and Mendell, 1992).

The ${ }_{\mathrm{NGN}} \mathrm{iSN}$ displayed robust membrane hyperpolarization during current-clamp recordings as shown in the example presented (Figure 3G). Quantification of the hyperpolarizationactivated current $\left(I_{h}\right)$, was obtained from measurement of the difference between the initial peak and the steady-state current during a $500 \mathrm{~ms}$ hyperpolarizing pulse of $55 \mathrm{pA}$ revealing a potential change of $-24.5 \pm 5.1 \mathrm{mV}(n=8$, 

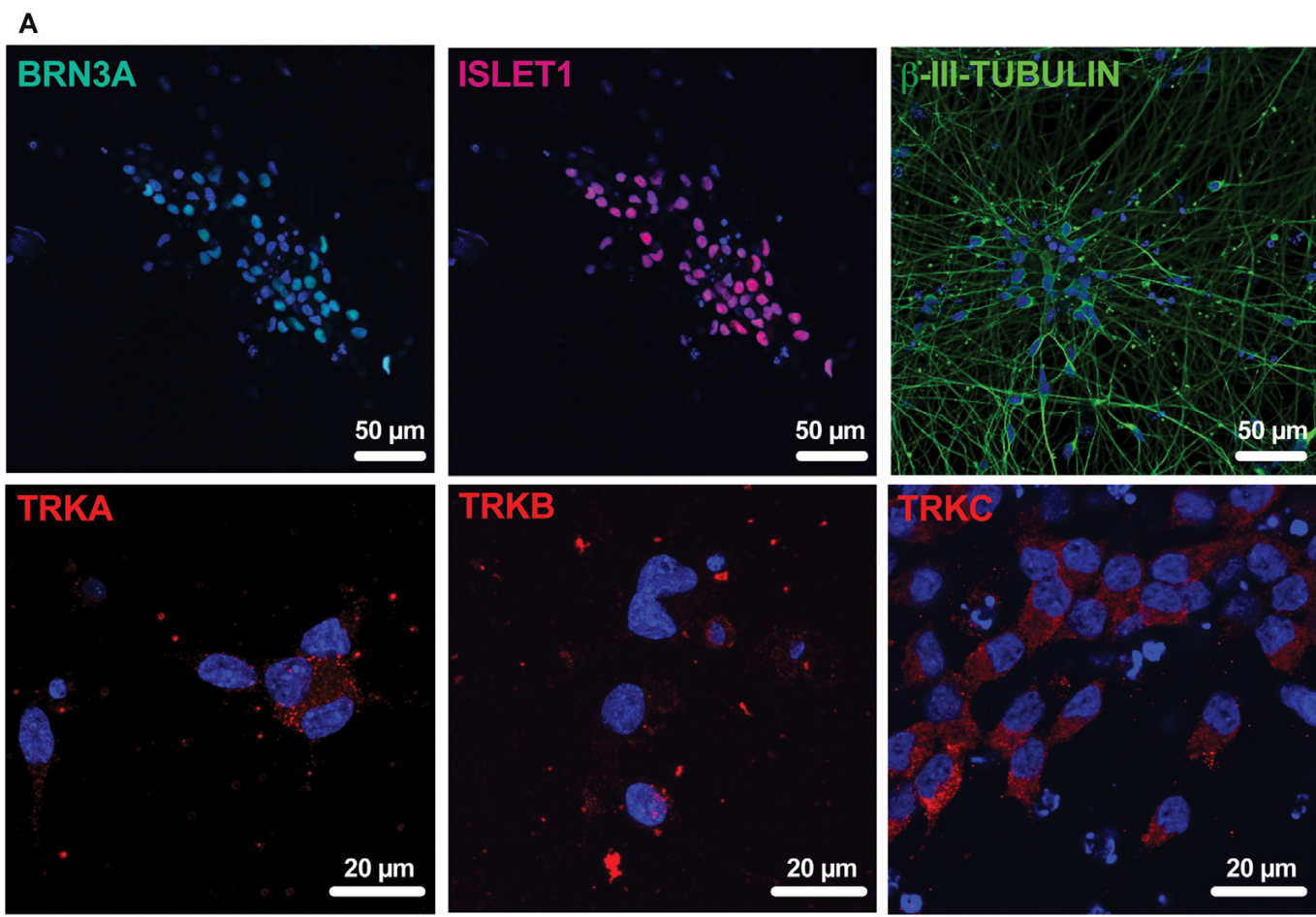

B

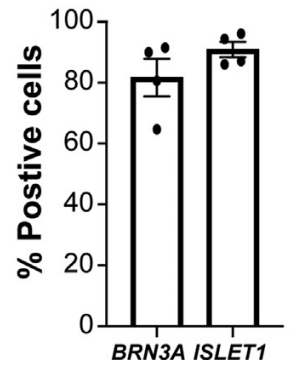

C

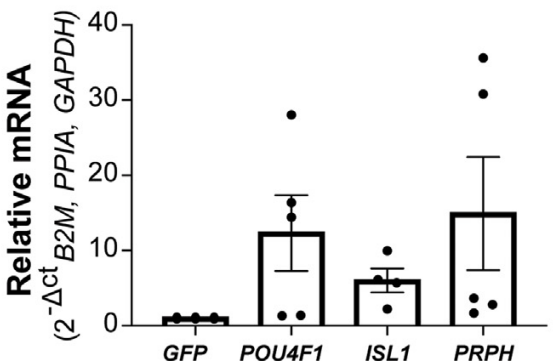

D

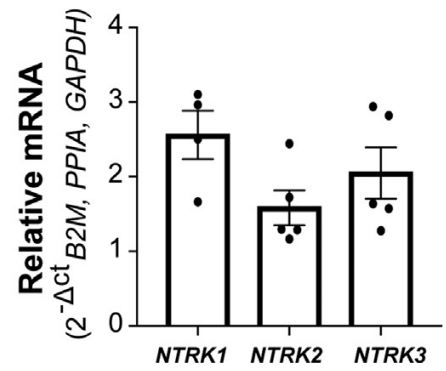

FIGURE 2 | Protein and mRNA expression of sensory neuron markers in ${ }_{\text {NGN2 }}$ iSNs. (A) ${ }_{\text {NGN2 }}$ iSN was positive for the protein expression of the neuronal marker B-III-TUBULIN (green) and the sensory neuron markers BRN3A (green), ISLET1 (red), TRKA (red), TRKB (red), and TRKC (red), nuclei shown in blue. (B) The percentage of neurons expressing either BRN3A or ISLET1, $n=4$ biological replicates, $>200$ cells counted per biological replicate. (C) Fold change in mRNA expression of POU4F1, ISL1, and PRPH normalized the GFP control. (D) Relative mRNA expression of NTRK1, NTRK2, NTRK3 (normalized to the house-keeping genes B2M, PPIA, and GAPDH), error bars presented as SEM, $n=5$ biological replicates.

Figure 3H). Hyperpolarization-activated cyclic nucleotide-gated (HCN) channels underlie $I h$ and are mediated by four major isoforms (HCN1-4). Accordingly, transcripts encoding all four family members were readily detected in our ${ }_{\mathrm{NGN} 2} \mathrm{iSN}$ cultures as evidenced by RT-qPCR (Figure 3I).

\section{Ionic Basis of $\mathrm{NGN}_{2} \mathrm{iSN}$ Excitability}

The generation and conduction of APs are critical to sensory neuron function. Therefore, the ion channels underpinning neuronal excitability are key players in sensory biology. In rodents, distinct voltage-gated ion channel constellations support the unique intrinsic properties of the major DRG sensory neuron classes (Zheng et al., 2019). In this study, we used voltage clamp to perform a detailed investigation of the whole-cell voltagedependent $\mathrm{Na}^{+}, \mathrm{K}^{+}$, and $\mathrm{Ca}^{2+}$ conductance components of the human ${ }_{\mathrm{NGN} 2} \mathrm{iSN} \mathrm{AP}$, as well as provide evidence of functional proton-activated currents $\left(I_{p H}\right)$ and its likely mediators.

\section{Voltage-Gated Sodium Currents}

Stem cell-derived neuronal cultures, such as those generated in this study, consist of an intricate network of cell bodies interconnected via long processes (Figure 1A, $\mathrm{NGN}_{2} \mathrm{iSN}$ ). In these networks, poor space clamp hinders control of $I_{\mathrm{Na}}$ from intact neurons, a challenge not experienced with primary DRG neurons, which are trimmed of long processes upon extraction and dissociation. Neuronal cell geometry affects the quality of 

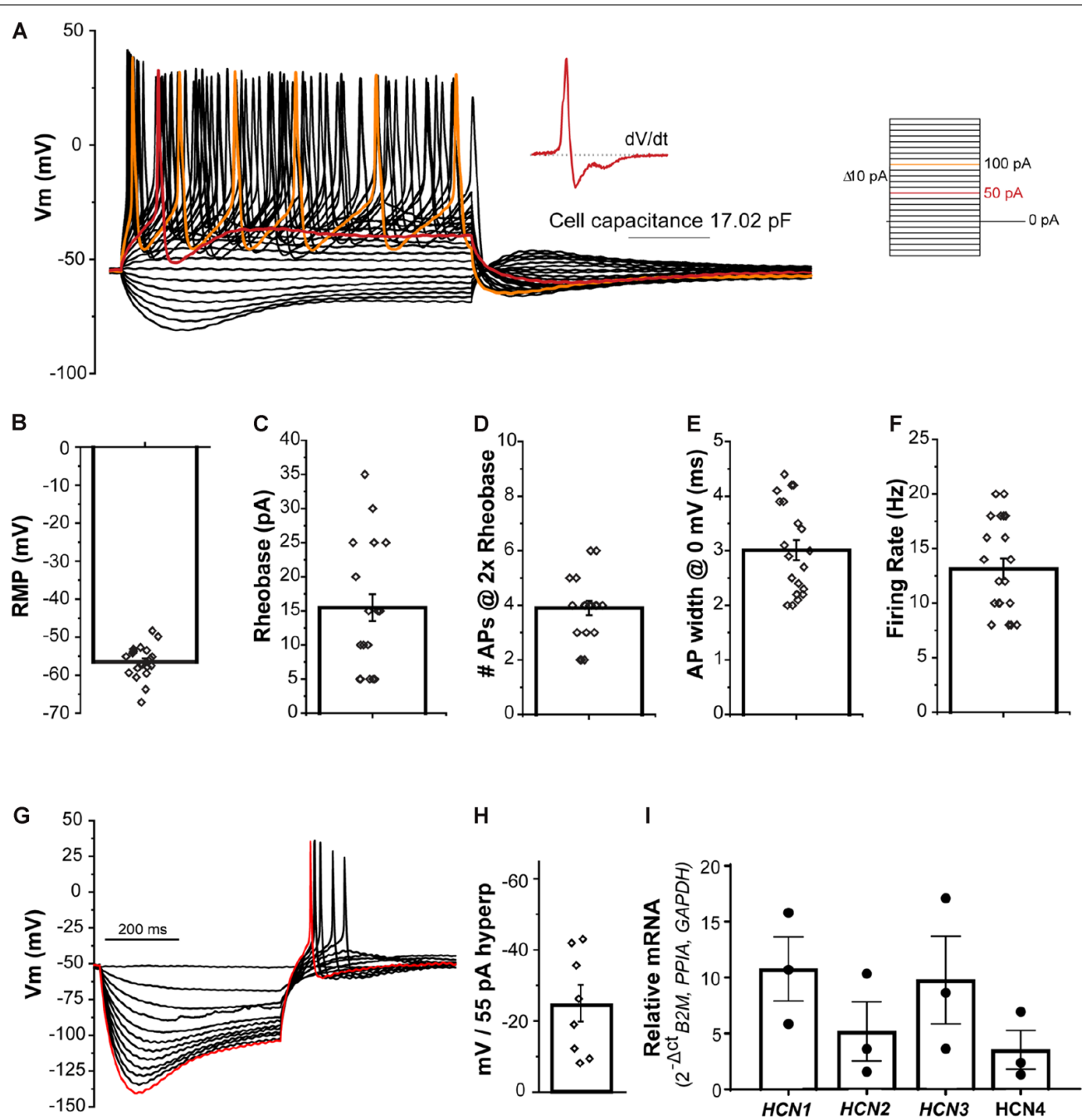

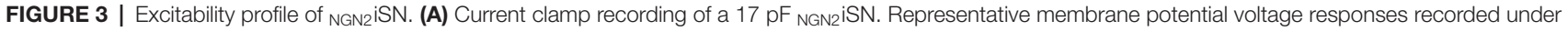
current-clamp conditions elicited by progressive current injections (from -60 to $+180 \mathrm{pA}, \Delta 10 \mathrm{pA}, 500 \mathrm{~ms}$ ). Traces corresponding to rheobase and $2 \times$ rheobase are highlighted in red and orange, respectively. Inset: first derivative of the trace at rheobase. (B-F) Summary of excitability properties: resting membrane potential

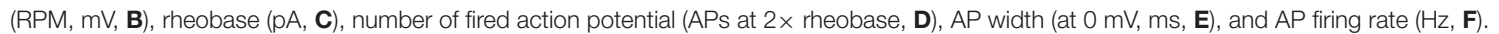

(G) Potassium-channel mediated membrane hyperpolarization upon current injections (0 to $-55 \mathrm{pA}, \Delta 5 \mathrm{pA}, 500 \mathrm{~ms})$. The membrane potential traces in response to $-55 \mathrm{pA}$ current injection is highlighted in red. (H) Bar graph showing $\mathrm{mV}$ change in peak vs. steady-state current amplitude after a $55 \mathrm{pA}$ hyperpolarizing pulse. (I) Relative mRNA expression of HCN1-4 transcripts detected in NGN2 iSN (normalized to the house-keeping genes B2M, PPIA, and GAPDH), error bars presented as SEM, $n=3$ biological replicates.

electrophysiological recordings. The axon and neurites constitute different compartments from the neuron's soma; hence, the voltage clamp of the cell body can fail to provide homogenous control of the membrane potential throughout the whole cell. Whole-cell currents from intact neurons under somatic voltage clamp contain a mixture of $I_{\mathrm{Na}}$ from the cell body and axial current from escaped axonal spikes. We used the method described by Milescu et al. (2010) to inactivate axonal sodium channels as a means to isolate and adequately control somatic $I_{N a}$ from the axial current thus providing a more accurate characterization of $I_{\mathrm{Na}}$.

Sensitivity to inhibition by the pufferfish alkaloid, tetrodotoxin (TTX), distinguishes two pharmacological families of voltage-dependent sodium channels (Navs). TTX sensitive (TTX-S) are those Nav isoforms blocked by low nM TTX whereas the TTX resistant (TTX-R) are represented by Nav isoforms inhibited by micromolar TTX exposure. The representative $I_{\mathrm{Na}}$ families shown in Figure 4 were elicited by the pulse protocol described in the inset and were recorded in the absence (Figure 4A) and presence of $300 \mathrm{nM}$ TTX (Figure 4B). We determined the contribution of each Nav-mediated component to the available total $I_{\mathrm{Na}}$ from $\mathrm{NGN}_{2} \mathrm{iSNs}$ in the absence (control) and after exposure to $300 \mathrm{nM}$ TTX. Ensemble current density estimated from peak $I_{N a}$ in response to a depolarizing pulse to $-10 \mathrm{mV}$ (red traces in Figures 4A,B) provides an estimate of the total $I_{\mathrm{Na}}$ 
A

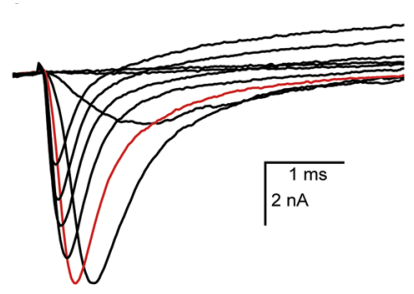

B
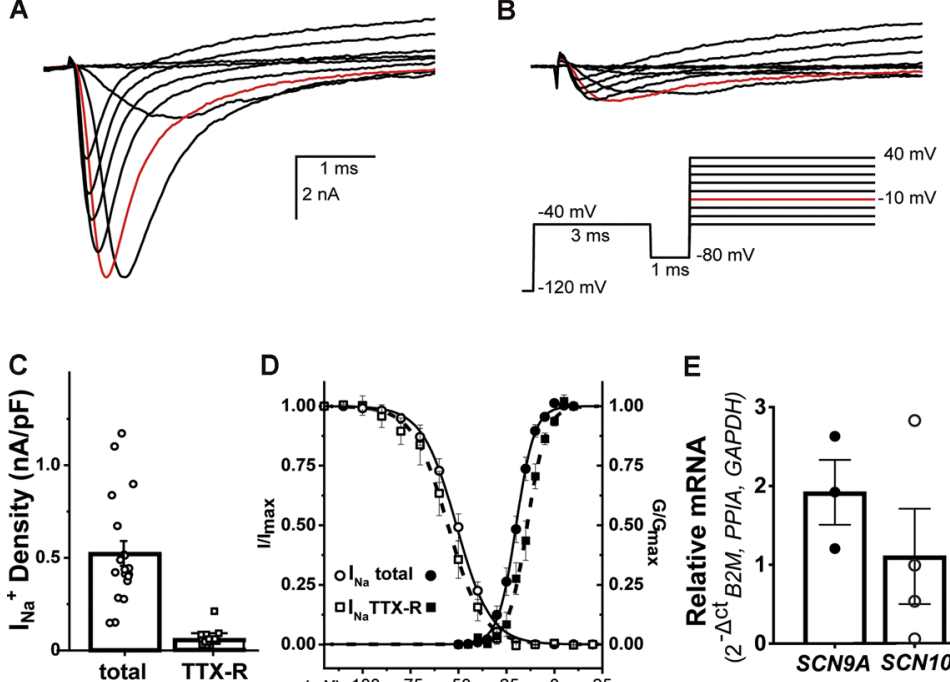

D

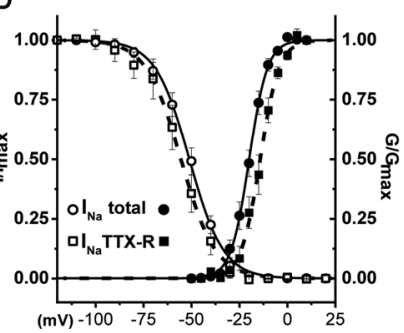

E

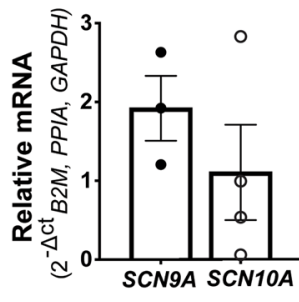

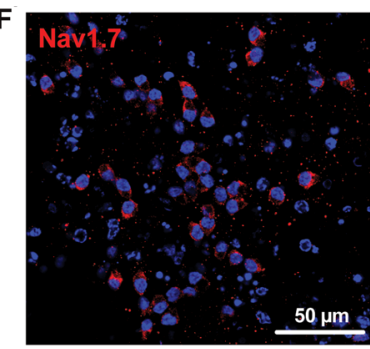

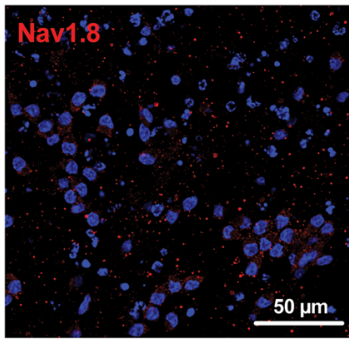

FIGURE 4 | Voltage-gated sodium currents in ${ }_{N G N 2}$ iSN. Sodium currents $\left(I_{N a}\right)$ from ${ }_{\text {NGN2 } 2}$ iSN are predominantly mediated by $T$ TX-S Nav channels. $(\mathbf{A}, \mathbf{B})$ Representative $/ /_{\mathrm{Na}}$ current traces in control $\mathbf{( A )}$ and the presence of $300 \mathrm{nM} T \mathrm{TX}(\mathbf{B})$ elicited by the voltage protocol shown in the inset. (C) NGN2 iSN sodium current $\left(I_{\mathrm{Na}}\right)$ density upon depolarization to $-10 \mathrm{mV}$ (highlighted in red in $\mathbf{A}$ and $\mathbf{B}$ ) calculated from peak total and TTX-R $I_{\mathrm{Na}}$ current in $\mathrm{nA} / \mathrm{pF}$. (D) $\mathrm{Voltage-}$ dependence of activation (filled symbols) and steady-state inactivation (SSI; empty symbols) of the total (•, O) and TTX-R (300 nM TTX, $\mathbf{\square}$, $\square$ ) components of $I_{\text {Na }}$. (E) Relative mRNA expression of SCN9A and SCN10A transcripts in NGN2 iSN (normalized to the house-keeping genes B2M, PPIA, and GAPDH), error bars presented as SEM, $n=3-4$ biological replicates. (F) Immunoreactivity against human Nav1.7 and Nav1.8 antibodies.

available in $\mathrm{NGN}_{2} \mathrm{iSNs}$ resulting in an average current density of $536.0 \pm 54.0 \mathrm{pA} / \mathrm{pF}$. The same measurements in the presence of $300 \mathrm{nM}$ TTX revealed that $\sim 13 \%$ of the total $I_{\mathrm{Na}}$ remains, which is equivalent to a current density of $68.4 \pm 10.5 \mathrm{pA} / \mathrm{pF}$ that is carried by TTX-R Nav channels in ${ }_{\mathrm{NGN}_{2}}$ iSNs $(n=19$, Figure 4C). Peak current amplitude from $I_{N a}$ families in the absence (Figure 4A) and presence of $300 \mathrm{nM}$ TTX (Figure 4B) were plotted as a function of the stimulus potential to determine the voltage-dependence of activation of the total $\left(I_{\text {total }}\right)$ and TTX-R ( $I_{\mathrm{TTX}-\mathrm{R}}$ ) components of ${ }_{\mathrm{NGN} 2} \mathrm{iSNs} I_{\mathrm{Na}}$ (Figure 4D). The total $I_{\mathrm{Na}}$ activates with a half-activation potential $\left(V_{0.5}\right)$ of $-19.9 \pm 0.2 \mathrm{mV}(\bullet, n=23$, Figure 4D), whereas the TTX-R component does so with a $V_{0.5}=-14.2 \pm 0.3(\boldsymbol{\square}, n=15$, Figure 4D). SSI curves were generated by a $500 \mathrm{~ms}$ pre-pulse to potentials from -120 to $+20 \mathrm{mV}$, in $10 \mathrm{mV}$ increments $\left(V_{\mathrm{h}}-80 \mathrm{mV}\right.$ ), followed by a test pulse to $0 \mathrm{mV}$ (Figure 4D). Peak $I_{\mathrm{Na}}$ amplitude measured upon the test pulse gauge the degree of Nav channel inactivation at the pre-pulse potentials. The bulk of the $I_{N a}$ inactivates with a $V_{0.5}=-51.1 \pm 0.3 \mathrm{mV}$ ( $\bigcirc, n=26$, Figure 4D) whereas the TTX-R $I_{\mathrm{Na}}$ in $\mathrm{NGN}_{2} \mathrm{iSN}$ displays an apparent SSI- $V_{0.5}$ of $-55.5 \pm 0.4 \mathrm{mV}(\square, n=16$, Figure 4D).

At the molecular level, we explored SCN9A and $S C N 10 A$ transcripts and their protein products Nav1.7 and Nav1.8 channels due to their apparent specific expression in sensory neurons. NGN2iSN had a high abundance of SCN9A and Nav1.7 expression, likely representing the TTX-S current and a lower proportion of SCN10A mRNA and Nav1.8 protein expression, consistent with a small contribution of the TTX-r current (Figures 4E,F).

\section{Voltage-Gated Potassium Currents}

Potassium currents $\left(I_{K}\right)$ are crucial regulators of neuronal excitability and homeostasis as they contribute to the RMP and membrane repolarization, thus they modulate the shape, duration, and frequency of APs. Voltage activated $\mathrm{K}^{+}(\mathrm{Kv})$ channels constitute the most diverse family with $40 \mathrm{Kv}$ members organized in 12 subfamilies which can make physiological identification of ion channels in neurons challenging (FinolUrdaneta et al., 2020). Yet, the properties and pharmacology of $I_{K}$ provide valuable information about sensory neuron functional subtypes (Giacobassi et al., 2020). To date, little information is available about the potassium channels expressed in stem cell-derived sensory neurons. A direct study by whole-cell patchclamp recordings from ${ }_{\mathrm{NGN} 2}$ iSNs was used to characterize its biophysical properties and their constituent ion channels.

Upon establishing the whole-cell configuration, cells were held at a $V_{\mathrm{h}}$ of $-80 \mathrm{mV}$. Typical whole-cell depolarizationactivated $I_{K}$ recordings are shown in Figures 5A,B. The whole-cell $I_{K}$ from ${ }_{\mathrm{NGN} 2} \mathrm{iSN}$ somatic recordings were large in virtually all cells recorded with patch electrodes of 2-4 M $\Omega$ resistance, yielding significant outward currents with peak amplitudes of $\sim 1.5 \mathrm{nA}$ (at $+20 \mathrm{mV}$, highlighted in red in Figure $5 \mathbf{A}) . I_{K}$ density was estimated from peak $I_{K}$ recorded at $+20 \mathrm{mV}$ normalized to the cell capacitance resulting in an average $I_{K}$ density of $124.5 \pm 11.7 \mathrm{pA} / \mathrm{pF}(n=29$, Figure 5C). A pre-pulse step to $-60 \mathrm{mV}$ or $-120 \mathrm{mV}$ (inset in Figure 5A) was implemented to differentiate between slow inactivating (delayed rectifier) and fast inactivating (A-type) $I_{K}$ components, respectively. However, within the same ${ }_{\mathrm{NGN} 2} \mathrm{iSN}$, currents elicited by either protocol had 

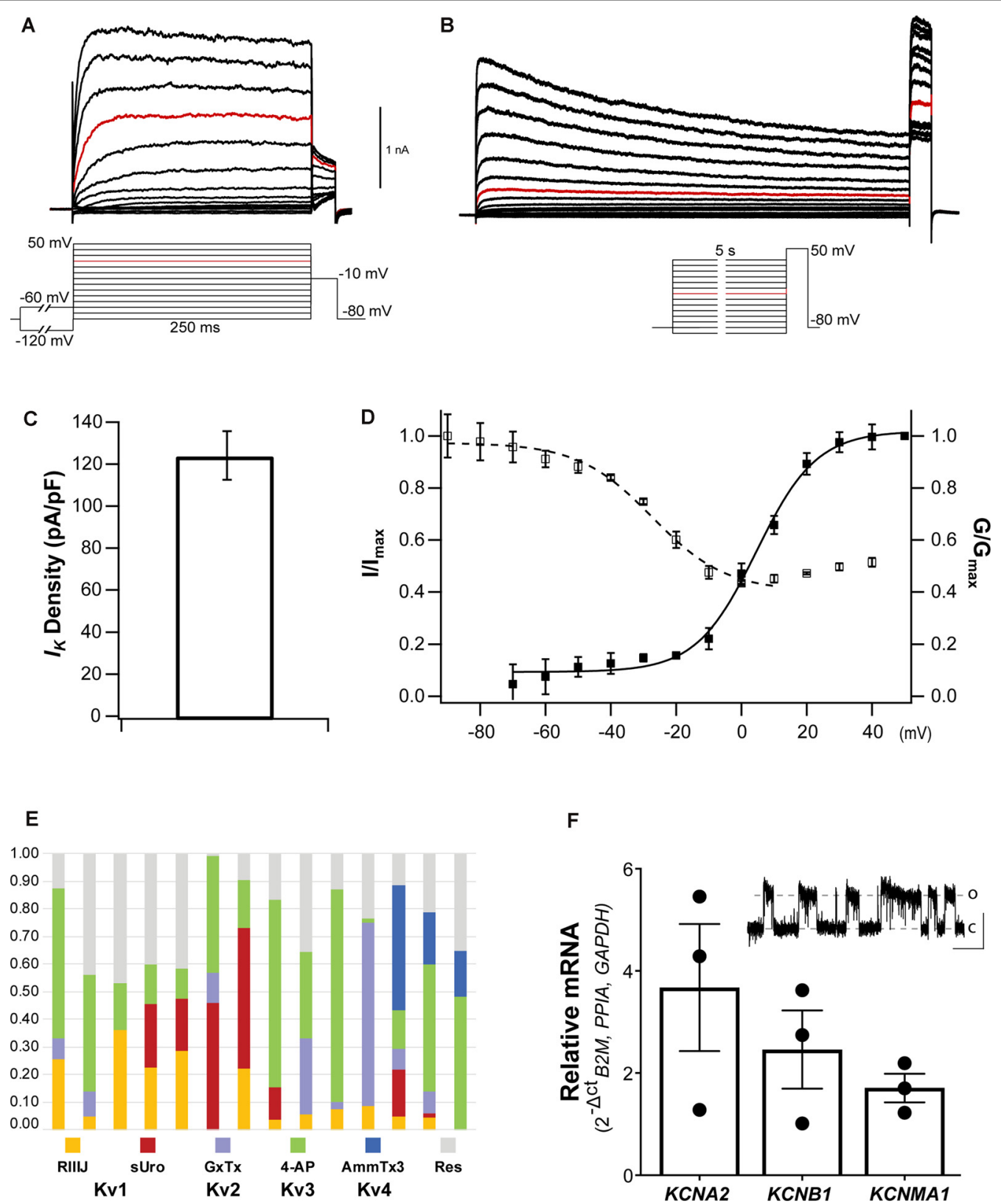

FIGURE 5 | Voltage-gated potassium currents in ${ }_{\text {NGN2 }}$ iSN. (A,B) Representative delayed rectifier potassium currents $\left(I_{K}\right)$ recorded in a $14.56 \mathrm{pF}$ cell upon standard

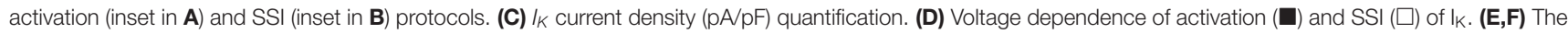
molecular identity of $\mathrm{NGN}_{2} \mathrm{iSN} I_{K}$. (E) Stacked bar plots of 14 different ${ }_{\mathrm{NGN} 2}$ iSNs showing the fraction of total outward $I_{K}$ current inhibited by inhibitors of KV1 ( $300 \mathrm{nM}$ RIIIJ and $10 \mathrm{nM}$ Urotoxin), Kv2 ( $300 \mathrm{nM}$ GxTx), Kv3 ( $100 \mu \mathrm{M}$ 4-AP), and Kv4 ( $1 \mu \mathrm{M}$ AmmTx3) channels. The fraction of $I_{K}$ remaining in the presence of all inhibitors is shown in gray ( Res). (F) Relative mRNA expression of the transcripts encoding the major Kv channels mediating $/ k$ in sensory neurons (KCNA2, KCNB1, and KCNMA1). The inset displays cell-attached single-channel recordings of a large conductance potassium channel consistent with the functional expression of $\mathrm{BK}\left(\sim 500 \mathrm{nM}\left[\mathrm{Ca}^{2+}\right]_{\text {, }}\right.$, pulse to $+80 \mathrm{mV}$, scale bars: 4 pA, $500 \mathrm{~ms}$, o, open; c, closed) .

indistinguishably slow kinetics suggesting a low contribution from fast inactivating $\mathrm{Kv}$ channels to the total $I_{K}$ in these cells. Thus, under our experimental conditions, the overwhelming majority of the elicited $I_{K}$ (Figure 5A) displayed characteristics consistent with those mediated by delayed rectifier Kv channels.
The voltage dependence and kinetics of activation and inactivation of ${ }_{\mathrm{NGN} 2} \mathrm{iSN}$ somatic $I_{K}$ were determined using the protocols described in the insets below the example families of outward $I_{K}$ traces (insets under Figures 5A,B for activation and inactivation, respectively). Instantaneous current-voltage (I-V) curves were well-fitted with a single, first-order Boltzmann 
function, yielding a mean $V_{0.5}$ of $4.6 \pm 1.1 \mathrm{mV}$ and slope factor $(k a)$ of $9.2 \pm 0.9(\boldsymbol{\square}, n=16$, Figure $5 \mathrm{D})$. Kv mediated currents were observed at potentials above $-60 \mathrm{mV}$ (Figure 5D), however, the threshold for voltage-activated $I_{K}$ was around $-30 \mathrm{mV}$. $\mathrm{NGN}_{2} \mathrm{iSN}$ rapid and voltage-dependent $I_{K}$ activation kinetics are well described by a single exponential function. Thus, the mean activation time constant for a voltage step from $V_{\mathrm{h}}$ to $+20 \mathrm{mV}$ was $8.1 \pm 2.4 \mathrm{~ms}(n=10)$ and decreased exponentially with voltage to $4.6 \pm 0.9 \mathrm{~ms}(n=10)$ for the step to $+60 \mathrm{mV}$. ${ }_{\mathrm{NGN} 2} \mathrm{iSN} I_{K}$ inactivation was slow and only appreciable during long stimulation protocol. The voltage dependence of inactivation was examined using the voltage protocol shown in the inset with marked current decay becoming evident during the $5 \mathrm{~s}$ long voltage steps (Figure 5B). A single Boltzmann function was used to describe the voltage dependence of inactivation of the major component as other contributors were too small to be accurately defined under our experimental conditions (Figure 5D). The midpoint of the voltage dependence of inactivation was $-27.4 \pm 2.2 \mathrm{mV}$ and slope factor $(k)$ of $11.3 \pm 2.02(\square, n=14$, Figure 5D).

Pharmacological assessment with selective inhibitors and venom-derived peptides constitutes a useful "dissection of the current" approach to identify individual mammalian voltagegated potassium channels. The contribution of various $\mathrm{Kv}$ channels to $I_{K}$ in $\mathrm{NGN}_{2} \mathrm{iSNs}$ is largely dependent on their kinetics and voltage-dependent activation and inactivation and therefore may have a maximal relative contribution to the whole-cell $\mathrm{Kv}$ currents at different time points and voltages. Fourteen individual $\mathrm{NGN}_{2} \mathrm{iSN}$ were surveyed for potential candidates underpinning $I_{K}$ by quantifying the fraction of current inhibited by a sequence of $\mathrm{Kv}$ channel inhibitors cumulatively applied at concentrations and order devised to enhance selective inhibition (Figure 5E). We began by applying $300 \mathrm{nM} \kappa$-conotoxin RIIIJ as this peptide is $\sim 10$-100-fold more potent against heteromeric than homomeric Kv1 channels and observed that 12 of 14 cells, displayed a RIIIJsensitive component (3-35\%) consistent with the expression of heteromeric channels containing Kv1.2 and likely Kv1.1 or Kv1.6 subunits (Cordeiro et al., 2019). The scorpion peptide Urotoxin $(10 \mathrm{nM})$ further blocked $2-46 \%$ of the outward $I_{K}$ evidencing functional expression of Kv1.2 homomeric channels in $50 \%$ of the tested cells $(7 / 14)$. The selective Kv2 channel spider toxin Guanxitoxin (300 nM) was used to evidence participation of Kv2 channels and observed a GxTx-sensitive component in $8 / 14 \mathrm{NGN}_{2}$ iSNs. A 4-aminopyridine (4-AP; $100 \mu \mathrm{M})$ sensitive component, ranging from $11 \%$ to $77 \%$ of the total $I_{K}$, was present in all cells tested highlighting the contribution of Kv3 channels that was evidenced by sequential application of this small molecule. As a final step, $1 \mu \mathrm{M}$ AmmTx3 identified the presence of Kv4 channels, likely associated with the accessory dipeptidyl peptidase-like proteins (DPP) 6 and 10, in 3/14 cells. Interestingly, the cumulative application of all these $\mathrm{Kv}$ channel inhibitors was not sufficient to eliminate all the voltage-dependent outward $I_{K}$ in most (13/14) ${ }_{\mathrm{NGN} 2}$ iSNs suggesting a substantial (9-47\%), yet unidentified, component of $I_{K}$ in human stem cell-derived sensory neurons.
RT-qPCR (or pharmacological) analysis of all potential potassium channel isoforms in our cultures was beyond the scope of this work. A scan for representative transcripts encoding for $\mathrm{Kv}$ channels commonly expressed in mammalian DRG sensory neurons revealed the presence of KCNA2, KCNB1, and KCNMA1 mRNA encoding for Kv1.2, Kv2.1, and BK channels, respectively in total ${ }_{\mathrm{NGN}}$ iSN RNA isolates (Figure 5F). The higher relative expression of KCNA2 transcripts is in agreement with the identification of RIIIJ- and Urotoxin-sensitive components; whereas the relative abundance of KCNB1 mRNA is consistent with the observed GxTx-sensitive component in ${ }_{\mathrm{NGN} 2}$ iSNs $I_{K}$.

In the whole-cell recording configuration, the presence of EGTA $(5 \mathrm{mM})$ in the patch pipette allows little to no contribution from $\mathrm{Ca}^{2+}$-activated potassium currents to the total $I_{K}$. Nevertheless, we observed the appearance of a $295.9 \pm 96.4 \mathrm{pS}($ at $+80 \mathrm{mV})$ potassium channel conductance with an open probability of 0.42 [intracellular $\left(\mathrm{Ca}^{2+}\right) \sim 500 \mathrm{nM}$ ] in cell-attached somatic patches of ${ }_{\mathrm{NGN} 2} \mathrm{iSN}$. These singlechannel recordings, observed in six out of 23 cells, are suggestive of the functional expression of BK channels and consistent with the detection of KCNMA1 transcripts in our neuronal cultures.

\section{Voltage-Gated Calcium Currents}

In dissociated rodent and human DRG neurons, $I_{C a}$ is mediated by different voltage-dependent $\mathrm{Ca}^{2+}$ channels (L-, N-, P/Q-, Rtype, and T-type channels), whose expression may be related to specific somatosensory cell types. In $\mathrm{NGN}_{2} \mathrm{iSNs}$, low (LVA or Ttype) and high voltage-activated (HVA, all other isoforms) $I_{C a}$ (Figure 6) could be readily identified in whole-cell recordings using external solutions designed to isolate $\mathrm{Ca}^{2+}$-mediated ionic currents. Representative LVA (gray) and HVA (black) $I_{\mathrm{Ca}}$ were elicited from a holding potential of $-90 \mathrm{mV}$ to a test pulse to -40 or $0 \mathrm{mV}$, to isolate LVA or HVA $I_{C a}$, respectively, for $100 \mathrm{~ms}$ at a frequency of $0.1 \mathrm{~Hz}$ (Figure 6A). The average current densities measured for LVA were slightly smaller than HVA (LVA $I_{C a}=13.4 \pm 1.5 \mathrm{pA} / \mathrm{pF}, n=10$; HVA $I_{C a}=18.8 \pm 1.5 \mathrm{pA} / \mathrm{pF}, n=10$, Figure 6B). The predominant channels carrying LVA $I_{C a}$ (Cav3.2/CACNA1H), and HVA $I_{C a}$ (Cav2.1/CACNA1A and Cav2.2/CACNA1B) in rodent DRG neurons were detected in human ${ }_{\mathrm{NGN}} \mathrm{iSN}$ by RT-PCR analyses (Figure 6C).

\section{Proton-Activated Currents}

Proton-activated currents have been historically associated with sensory neurons, its mediators the acid-sensing (ASIC1-4) and transient receptor potential vanilloid (TRPV) channels are well-established modulators of mechanosensation, nociception, and proprioception (Davis et al., 2000; Walker et al., 2003; Gu and Lee, 2010; Omerbašic et al., 2015). Therefore, we explored the activation of these ionic currents in the ${ }_{\mathrm{NGN} 2}$ iSN generated in this study by a drop in extracellular $\mathrm{pH}$ and their sensitivity to amiloride to distinguish between ASIC and TRPV1 mediated proton-sensitive currents.

Whole-cell proton-activated currents were elicited by rapidly decreasing the extracellular solution $\mathrm{pH}$ from 7.4 to $6.0\left(I_{p H 6.0}\right)$. Patch-clamp recordings of ${ }_{\mathrm{NGN} 2} \mathrm{iSNs}$ proton currents in control and in the presence of the heterotrimeric sodium $(\mathrm{ENaC})$ 

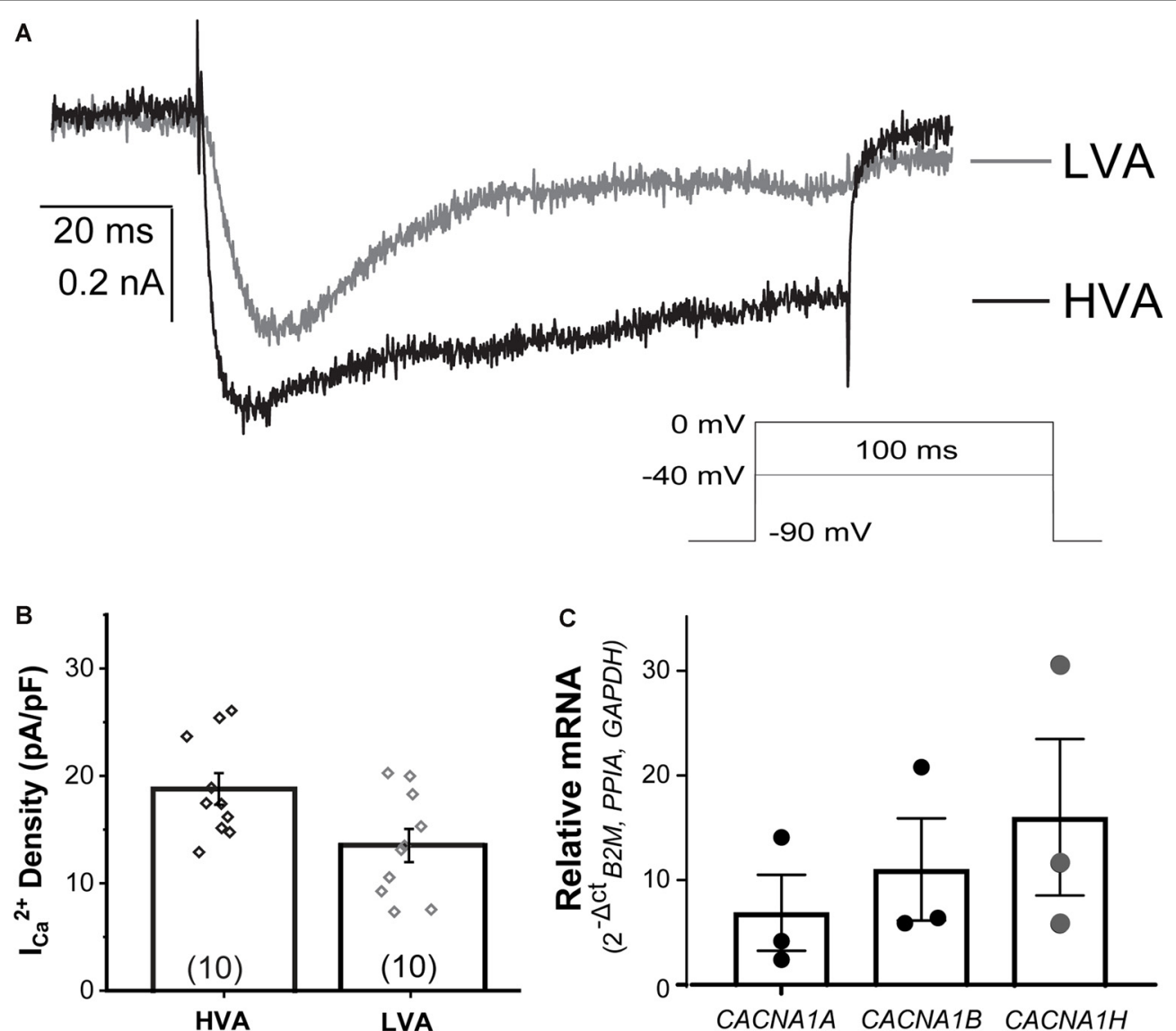

FIGURE 6 | Voltage-gated calcium currents $\left(I_{\mathrm{Ca}}\right)$ present in NGN2 $\mathrm{iSN}$. NGN2 $\mathrm{iSN} I_{\mathrm{Ca}}$ evidence contribution from high and low voltage-activated calcium channels (HVA and LVA, respectively). (A) Representative HVA at $0 \mathrm{mV}$ (black) and LVA at $-40 \mathrm{mV}$ (gray) $I_{\mathrm{Ca}}$ traces. Stimulation protocol is shown in the inset (100 ms Vh $-90 \mathrm{mV}$, $0.2 \mathrm{~Hz})$. (B) Bar graph summarizing $I_{\mathrm{Ca}}$ current density $(\mathrm{pA} / \mathrm{pF})$ of the HVA and LVA mediated components. (C) Relative mRNA expression of transcripts encoding the major calcium channels mediating HVA (CACNA1A, CACNA1B) and LVA (CACNA1H) in NGN2iSN (normalized to the house-keeping genes B2M, PPIA, and GAPDH), error bars presented as SEM, $n=3$ biological replicates.

channel blocker, amiloride, are shown in Figure 7A. The drop of extracellular $\mathrm{pH}$-induced transient inward currents whose maximal amplitude was used to determine the overall ${ }_{\mathrm{NGN} 2} \mathrm{iSN}$ $I_{p H 6.0}$ density resulting in $59.8 \pm 3.31 \mathrm{pA} / \mathrm{pF}(n=13$, Figure $7 \mathbf{B})$. In sensory neurons, proton activated current can be mediated by TRPV1 or any of the ASIC family channels. In the presence of $10 \mu \mathrm{M}$ amiloride, $\sim 15 \%$ of $I_{p H 6}$ remained (\% block $86.9 \pm 1.19 \%$, $n=6$, Figure 7 ) in the ${ }_{\mathrm{NGN}_{2}}$ iSNs tested consistent with relatively low abundance of TRPV1 protein and transcripts detected in our cultures by immunofluorescence and qPCR (Supplementary Figure 4). At the concentration used, amiloride fully inhibits ASIC channels whilst sparing TRPV1 thus suggesting a sizeable contribution of ASIC-mediated currents to the proton sensitive conductance in human ${ }_{\mathrm{NGN} 2}$ iSN cells.

\section{DISCUSSION}

This study describes the generation and functional characterization of human sensory neurons derived from hPSCs. We optimized a protocol that results in abundant

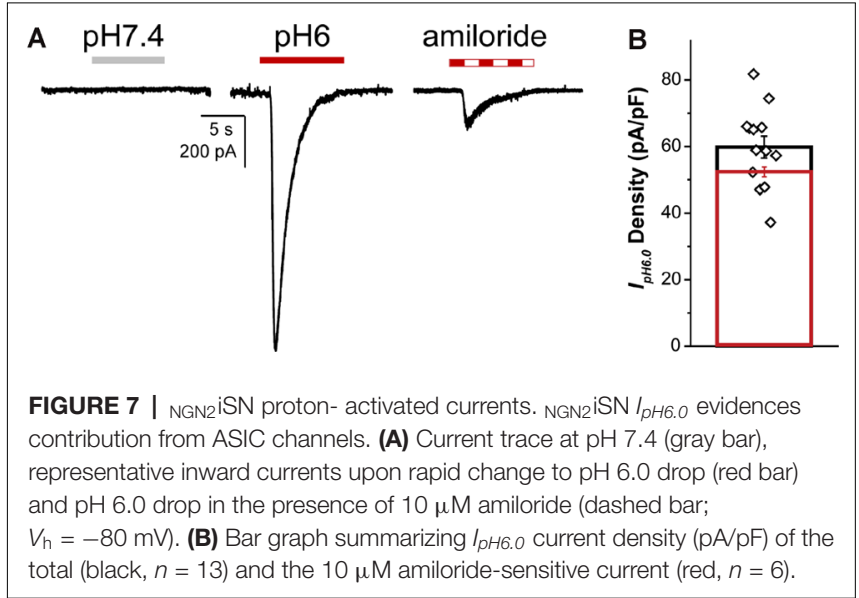

electrically excitable cells capable of firing regenerative APs. The biophysical and pharmacological properties of voltagedependent ionic currents in ${ }_{\mathrm{NGN} 2} \mathrm{iSNs}$ were investigated and 
related to those known to be present in mammalian primary sensory neurons. The present study represents the first detailed biophysical and pharmacological characterization of the different conductances supporting excitability in human sensory neurons and gives insights into the molecular identities of native ionic currents.

We have optimized the generation of functionally mature sensory neurons from hPSCs by enriching our cultures for $\mathrm{NC}$ cells and then inducing the expression of the transcription factor NGN2. NC enrichment was enhanced by plating whole neurospheres in the presence of the ROCK inhibitor Y-27632 instead of disaggregating the spheres into single cells. Plating whole spheres results in a significantly higher proportion of SOX10+ cells, a key marker for migratory cells, compared to the dissociation into single cells approach ( $66 \%$ vs. $19 \%$, respectively). The increased proportion of SOX10+ cells is attributed to the inhibition of the ROCK pathway, which was shown to promote the delamination and differentiation of $\mathrm{NC}$ cells from the developing neural tube (Groysman et al., 2008) and effectively increases the proportion of NC cells during hPSC differentiation (Hotta et al., 2009; Kim et al., 2015). Interestingly, SOX10+ cells appeared after just $24 \mathrm{~h}$ of neurosphere plating, whereas P75 ${ }^{\text {NTR }}$ positive cells were first observed after $48 \mathrm{~h}$ of plating. This is consistent with SOX10 expression spanning various stages of NC development (e.g., from the progression of NC precursors to migrating crest cells), with $\mathrm{P} 75^{\mathrm{NTR}}$ predominantly co-expressed with SOX10 in the migratory cells (McKeown et al., 2005; Betters et al., 2010; Curchoe et al., 2010). The expression of $\mathrm{PAX} 3 / 7$, which are transcription factors commonly expressed throughout NC development and specifically in pre-migratory and migratory NC cells (Basch et al., 2006; Betters et al., 2010; Maczkowiak et al., 2010; Murdoch et al., 2012), was also noted in the cultures at varying levels. Thus, we surmise that our $48 \mathrm{~h}$ cultures, which contain SOX10/P75 ${ }^{\mathrm{NTR}}$ positive cells, most likely represent migrating NC cells enriched culture in which the expression of NGN2 further drives the differentiation to functionally mature sensory neurons. Immunoreactivity towards key protein markers confirms the successful differentiation of sensory neurons following NGN2 expression verified by immunofluorescence confocal microscopy. A significant proportion of $\mathrm{NGN}_{2} \mathrm{iSNs}$ derived from the described protocol was positive for the pan-sensory neuron markers BRN3A and ISLET1 $(\sim 80-90 \%$ positive), which is notably higher than previous protocols not implementing NGN2 expression in NC cells $(\sim 5 \%$

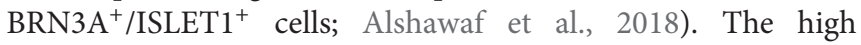
proportion of BRN3A expression throughout our cultures is qualitatively similar to previously described differentiation protocols inducing the expression of different combinations of transcription factors, such as BRN3A, NGN1, and NGN2 to generate sensory neurons. However, in contrast to previous reports (Blanchard et al., 2015, Schrenk-Siemens et al., 2015) we quantified BRN3A expression within the whole culture (e.g., without excluding non-neuronal cells). To address cellular heterogeneity within the culture dish and avoid potential bias, the number of $\mathrm{BRN}_{3} \mathrm{~A}^{+}$neurons was normalized to the total cell count within the dish. Consequently, the differentiation strategy presented in this work generated $82 \% \mathrm{BRN}^{2} \mathrm{~A}^{+}$cells, thus evidencing an enrichment for sensory neurons and a significantly low representation of non-neuronal phenotypes. Detection at the protein and transcript level, of the three major sensory neuron markers TRKA, TRKB, and TRKC in our sensory neuron cultures suggest that our differentiation protocol generates a heterogeneous population of sensory neurons, including nociceptors, mechanoreceptors, and proprioceptors. In general, in vitro stem cell differentiation cannot fully replicate all of the differentiation cues found in vivo, particularly in terms of establishing the proportion of cell types within a tissue or ganglia, such as the DRG. This is partly due to the challenge of replicating in vitro the timing of $\mathrm{NC}$ migration and differentiation that occurs in vivo, which gives rise to the different sensory neuronal populations. The heterogeneity of sensory subtypes found within the $\mathrm{NGN}_{2} \mathrm{iSN}$ cultures underscores the need for further work to delineate strategies aimed at enriching specific sensory subtypes and in-depth profiling of each of those subtypes. In this study, we used the transcription factor-mediated approach to direct crest differentiation to DRG sensory neurons. This approach can certainly be further developed to direct sensory neuronal differentiation to a specific phenotype/lineage if the specifying transcription factors for that lineage are known. Importantly, since the ${ }_{\mathrm{NGN}} \mathrm{iSN}$ cultures consist of heterogeneous sensory neuronal populations, these cultures represent an excellent model of human DRG neurons that can be used to further study the human sensory system and develop therapies for related sensory pathological conditions.

The use of molecular analyses, such as immunocytochemistry and RT-qPCR, are important in determining the success of differentiating hPSC to sensory neurons. However, the functional excitability profile of the generated neurons must be investigated to provide a reliable functional baseline for the model's applicability in the investigation of diseases or the effect of drugs on defined ion channels. Functional profiling of hPSC-derived sensory neurons has primarily been focused on protocols that generate nociceptors (Chambers et al., 2012; Young et al., 2014; Blanchard et al., 2015; Eberhardt et al., 2015; Wainger et al., 2015; McDermott et al., 2019; Meents et al., 2019; Schoepf et al., 2020), rather than a heterogeneous population of sensory neurons akin to a more physiologically relevant model in which diverse populations of sensory neurons coexist. In this study, we sought to profile the spontaneously resulting mixed population of DRG sensory neurons at the transcriptional and functional levels including a detailed description of ${ }_{\mathrm{NGN} 2} \mathrm{iSN}$ excitability patterns and their underlying voltage-dependent conductances.

Our ${ }_{\mathrm{NGN} 2} \mathrm{iSN}$ display phasic firing at relatively low current injections (average rheobase: $15 \mathrm{pA}$ ) that become tonic adapting AP firing of increasing frequency (max firing rate $13 \mathrm{~Hz}$ ) with subsequently stronger stimulation, but no spontaneous firing under our experimental conditions. The observed heterogeneous electrophysiological signatures include repetitive low-frequency firing in response to current injection, delayed firing with long latency to the first AP followed by repetitive spiking, various 
patterns of firing adaptation as well as cells with "humped" AP (Supplementary Figure 3). As the major determinant of neuronal excitability, the RMP of the $\mathrm{NGN}_{2}$ iSN generated with the described protocol had RMPs (Figure 3B, $-56.4 \pm 0.9 \mathrm{mV}$, $n=21$ ) comparable to those found in dissociated human sensory neurons from young adults (mean age 18.2 years old) in culture $(-62.4 \pm 2.0 \mathrm{mV}, n=133$; Two-tailed $P$-value $=0.2375$; Davidson et al., 2014). These features highlight the suitability of our $\mathrm{NGN}_{2} \mathrm{iSN}$ cultures for modeling human sensory neuron function, its pharmacological modulation, and pathophysiology at the population level rather than a specific subtype.

The excitability of the different modalities of sensory neurons is shaped by unique patterns of ion channel expression. HCN channels mediate the excitatory hyperpolarization-activated current often associated with the repetitive neuronal electrical activity (Sartiani et al., 2017). Current clamp recordings from $\mathrm{NGN}_{2} \mathrm{iSNs}$ displayed hallmark features of Ih such as a voltage sag during hyperpolarizing current injection followed by rebound excitation upon current termination (Mayer and Westbrook, 1983; Robinson and Siegelbaum, 2003; Poolos, 2004). Consistent with studies of $I h$ in rodent DRG neurons (Kouranova et al., 2008), voltage sag onset was slow, appeared at potentials negative to $-55 \mathrm{mV}$, and often led to AP firing upon rebound. The observed slow Ih onset and relative expression of HCN3 transcripts detected in the $\mathrm{NGN}_{2} \mathrm{iSN}$ cultures are consistent with the functional properties of slow-gating $\mathrm{HCN} 3$ channels recorded in recombinant systems (Stieber et al., 2005). Moreover, we observed HCN1 transcripts in our ${ }_{\mathrm{NGN}} \mathrm{iSN}$ cultures in agreement with recently published multiplex RNAscope in situ hybridization data from human DRG neurons of all sizes (Shiers et al., 2020). An in-depth biophysical characterization aided by pharmacological tools of human primary sensory neurons will warrant a better understanding of the homoand heteromeric $\mathrm{HCN}$ channels complement present in these cells.

The development of neuronal excitability involves the coordinated expression of different voltage-gated ion channels. To define the molecular basis of ${ }_{\mathrm{NGN} 2} \mathrm{iSN}$ excitability, we dissected the major voltage-dependent conductances underlying the AP in human sensory neurons. We focused on the two major classes of voltage-dependent $I_{\mathrm{Na}}$ studied in sensory neurons, which are broadly represented by the low threshold, rapidly activating and inactivating TTX-S Nav channels, and by the high threshold, more slowly activating and inactivating TTX-R Nav isoforms. We observed a healthy complement of $I_{\mathrm{Na}}$ in $\mathrm{NGN}_{2} \mathrm{iSN}$ mediated largely ( 90\%) by TTX-S channels. Accordingly, the biophysical properties of the total $I_{\mathrm{Na}}$ (activation $V_{0.5}-19.9 \mathrm{mV}$, SSI $V_{0.5}-51 \mathrm{mV}$ ) closely approximate those of human DRG TTX-S $I_{\mathrm{Na}}$ (TTXs activation $V_{0.5}-15 \mathrm{mV}$, SSI $-58 \mathrm{mV}$, Zhang et al., 2017). The voltage dependence of activation of ${ }_{\mathrm{NGN} 2} \mathrm{iSN}$ TTX-R $I_{\mathrm{Na}}$ is similar to that of dissociated human primary DRG neurons ( ${ }_{\mathrm{NGN} 2} \mathrm{iSN}$ TTX-R $I_{\mathrm{Na}}$ activation $V_{0.5}-14.2 \mathrm{mV}$ vs. human DRG TTX-R activation $V_{0.5}-10 \mathrm{mV}$, Zhang et al., 2017). However, the SSI of the TTX-R $I_{\mathrm{Na}}$ recorded in $\mathrm{NGN}_{2} \mathrm{iSN}$ is significantly left-shifted (SSI $V_{0.5}-55 \mathrm{mV}$ ) in comparison to human DRG reported by Zhang et al. (2017; SSI $V_{0.5}$ $-20 \mathrm{mV})$.

The TTX-S Nav1.7 channel is typically associated with all sensory neuron subtypes and SCN9A mRNA is reported in virtually all neurons of human DRG (Ho and O'Leary, 2011; Haberberger et al., 2019; Shiers et al., 2020). We detected SCN9A transcripts in total RNA isolates and immunoreactivity with the Nav1.7 antibody, whilst observed low immunoreactivity towards Nav1.8, which is typically found in nociceptors. These, together with comparatively low TRKA staining in our cultures suggests that our protocol does not generate a high proportion of nociceptors and that other TTX-S Nav channels such as Nav1.6 and Nav1.1, which are abundantly detected in rodent mechano- and proprio-sensory neurons (Zheng et al., 2019), are also likely mediating the $\mathrm{NGN}_{2}$ iSN TTX-S $I_{\mathrm{Na}}$. A small but readily observable TTX-R $I_{N a}$ component mediated by Nav1.8 was identified in these cultures. In human DRG, Nav1.8 immunoreactivity has been reported in several classes of sensory neurons, such as nociceptors and rapidly adapting $\mathrm{A} \beta$ low threshold mechanoreceptors (A $\beta$-LTM) surrounding hair follicles in human skin (Coward et al., 2000; Shields et al., 2012). Here, we used cell morphology and apparent membrane fitness to guide our patch-clamp characterization, resulting in a potential bias towards comparatively larger neurons in the cultures which could imply a higher representation of mechanosensory neurons in our recordings.

Voltage-dependent potassium currents have a pivotal role in the regulation of neuronal firing properties, such as the $\mathrm{RMP}$, AP firing threshold, as well as AP shape and frequency. Importantly, differential modulation of $I_{K}$ can determine the response modality of their hosting neurons (González et al., 2017). The sheer abundance and diversity of $\mathrm{K}^{+}$channels are a reflection of their relevance but also a daunting obstacle in their study at the physiological level (Cordeiro et al., 2019; Finol-Urdaneta et al., 2020; Giacobassi et al., 2020). A significant body of work on the various voltage-gated $I_{K}$ present in rodent DRGs exists, yet little, if anything, is known regarding the various $\mathrm{K}^{+}$conductances present in human sensory neurons. This work on human ${ }_{\mathrm{NGN}} \mathrm{iSN}$ neurons provides a starting point to explore this important family of channels. Based on current kinetics, neuronal $I_{K}$ is mediated by fast inactivating $\left(I_{K A}\right)$ and slow inactivating delayed rectifying $\left(I_{K D R}\right)$ $\mathrm{Kv}$ channels. Hyperpolarizing pre-pulse protocols failed to demonstrate the presence of fast inactivating currents (not shown), therefore, we conclude that most of the $\mathrm{NGN}_{2} \mathrm{iSN}$ neurons analyzed here express large delayed rectifying outward currents. Several components of $I_{K}$ can be differentiated in patch-clamp recordings. Given the similarities in current kinetics and their activation/inactivation thresholds, we performed a pharmacological survey to approximate major molecular families mediating Kv currents and detected selected channel transcripts by RT-qPCR. All neurons recorded had a 4-AP sensitive component, consistent with the expression of slow inactivating members of the Kv3 family, whose principal physiological role involves the regulation of neurotransmitter release (Kaczmarek and Zhang, 2017). Interestingly, the transient presence of Kv3 family channels at the earliest stages of the rat and chicken 
nervous system development suggests that these channels may have a developmental role apart from those regulating firing rates and neurotransmitter release in adult neurons (Kaczmarek and Zhang, 2017). Future work warrants investigation of the relevance of Kv3 channel isoforms expression in identified subpopulations of human DRG neurons.

Sensitivity to conotoxin $\kappa$-RIIIJ (300 nM) in $\sim 85 \%$ of cells tested revealed the functional expression of heteromeric Kv1.2-Kv1.1/Kv1.6 channels suggesting parallels with mouse L1 proprioceptive neurons (Giacobassi et al., 2020). Block by the Kv1.2-selective scorpion peptide Urotoxin was used to isolate the presence of homomeric Kv1.2 channels (Luna-Ramirez et al., 2020), which could be seen in half of the neurons screened consistent with abundant detection of KCNA2 mRNA in our cultures. Clusters of Kv1.2 channels have been identified at the juxtaparanodes in co-cultures of human-induced pluripotent stem cell-derived sensory neurons and rat Schwann cells (Clark et al., 2017) as well as in rodent L2 A $\delta$-LTMR neurons (Giacobassi et al., 2020).

Detection of KCNB1 transcripts by RT-qPCR and inhibition by spider peptide Guanxitoxin (Liu and Bean, 2014) are indicative of the expression of Kv2 channel family members in $57 \%$ of cells. In humans, KCNB1 transcripts are present in $85.4 \%$ of small and large diameter DRG sensory neurons (Shiers et al., 2020), whereas Kv2 channels are expressed in all modalities of rodent mechanoreceptors (Zheng et al., 2019). In contrast to the widespread expression of the Kv4 family in rodent DRG (NaPhuket and Covarrubias, 2009; Zheng et al., 2019), we observed Kv4 channel inhibition by AmmTx3 (Pathak et al., 2016) only in a small subset of the ${ }_{\mathrm{NGN} 2} \mathrm{iSNs}$ analyzed. Taken together, we found considerable variation in $\mathrm{Kv}$ currents within our cultures yet $I_{K D R}$ was the dominant outward current component of human NGN2 2 SN $I_{K}$.

Each DRG neuronal subtype exhibits a unique complement of voltage-gated ion channels that shapes the transduction of sensory information. Voltage-gated $\mathrm{Ca}^{2+}$ (Cav) channels activation enables the exocytosis of neurotransmitter-filled synaptic vesicles hence they are critically important to neuronal sensory function (Park and Luo, 2010). Cav channel currents mediated by the LVA and HVA Cav channel families were recorded electrophysiologically, as well as their encoding mRNAs NGN2 iSNs. Transcripts encoding the $\alpha$-subunits of (HVA) $\mathrm{P} / \mathrm{Q}$ isoform Cav2.1 and N-type Cav2.2 HVA channels were identified, consistent with transcriptomic analyses of mouse sensory neurons (Zheng et al., 2019). Cav channel function in the human DRG remains unexplored, however, consistent with our RT-qPCR data, expression of CACNA1B mRNA (Cav2.2) has been reported in dissociated human DRGs (Castro et al., 2017). NGN2iSN display robust LVA $I_{C a}$ akin to those mediated by the T-type calcium channel family. The magnitude of LVA currents in ${ }_{\mathrm{NGN}} \mathrm{iSN}$ and the relatively low abundance of TRPV1 signal detected (immunofluorescence and qPCR) here suggest a parallel between our cultures and previously described subpopulations of capsaicin insensitive DRG and trigeminal neurons from adult mice (Pearce and Duchen, 1994; Borgland et al., 2001; Fioretti et al., 2011). It is of note, however, that capsaicin inhibits LVA calcium channels indirectly via calcium influx through
TRPV1 (Comunanza et al., 2011) in DRG neurons and in HEK293 cells co-expressing Cav3.1 and TRPV1 (McArthur et al., 2019). Furthermore, capsaicin also directly inhibits all T-type channel isoforms with $\mathrm{IC}_{50} \mathrm{~s} \sim 20 \mu \mathrm{M}$ (McArthur et al., 2019) thus suggesting that the magnitude of T-type calcium currents may be underestimated in the presence of capsaicin.

RT-qPCR analysis of $\mathrm{NGN}_{2} \mathrm{iSN}$ evidence expression of transcripts encoding for TRKB receptor (encoded by NTRK2) and the T-type Cav3.2 (encoded by CACNA1H). The latter is regarded as a selective marker of $\mathrm{A} \delta$ - and C-low-threshold mechanoreceptors (LTMRs) innervating mouse skin hair follicles where Cav3.2 activation regulates light-touch and pain perception (Rutlin et al., 2014; François et al., 2015) supporting the generation of low threshold mechanoreceptors, as well as other sensory neurons using the hereby described protocol.

ASICs have a primary role in monitoring changes in extracellular acidosis caused by inflammation and injury and are also involved in the detection and transduction of mechanical stimuli (Price et al., 2000; Page et al., 2005; Deval et al., 2008; Gu and Lee, 2010; Geffeney and Goodman, 2012; Lin et al., 2016; Cheng et al., 2018). ASIC expression has been detected in nociceptors, mechanoreceptors, and proprioceptors, albeit with different subunit expression patterns depending on the sensory neuron subtype (Flegel et al., 2015; Usoskin et al., 2015; Lin et al., 2016; Papalampropoulou-Tsiridou et al., 2020). Thus, we investigated the presence of proton-sensitive currents and the expression of ASICs in our $\mathrm{NGN}_{2}$ iSNs. A rapid decrease in $\mathrm{pH}$ elicited large inward currents, which was then inhibited by low concentrations of the pan-ASIC blocker, amiloride, in our ${ }_{\mathrm{NGN} 2} \mathrm{iSNs}$. This functional data indicate the presence of functional ASICs in ${ }_{\mathrm{NGN}} 2 \mathrm{iSNs}$. Interestingly, $\sim 10 \%$ of the proton-activated current was insensitive to amiloride suggesting the contribution from other proton-gated channels.

\section{CONCLUSION}

This study describes the differentiation of hPSCs into functional sensory neurons, via a combined small molecule and transcription factor expression approach. The $\mathrm{NGN}_{2}$ iSNs expressed sensory neuron markers and exhibited robust AP firing supported by voltage-gated $\mathrm{Na}^{+}, \mathrm{K}^{+}$, and $\mathrm{Ca}^{2+}$ conductances Our characterization of stem cell-derived sensory neurons sheds light on the molecular basis of human sensory physiology and highlights the suitability of using hPSC-derived sensory neurons for modeling human DRG development and their potential in the study of human peripheral neuropathies and drug therapies.

\section{DATA AVAILABILITY STATEMENT}

The raw data supporting the conclusions of this article will be made available by the authors, without undue reservation.

\section{ETHICS STATEMENT}

The studies involving human participants were reviewed and approved by University of Wollongong Human Ethics Committee. Written informed consent for participation was not 
required for this study in accordance with the national legislation and the institutional requirements.

\section{AUTHOR CONTRIBUTIONS}

AJH, JRM, RKF-U, and MD conceived and designed the study. AJH performed the cell culture and molecular profiling of the $\mathrm{NGN}_{2} \mathrm{iSN}$, analyzed expression data and presentation. JRM and RKF-U performed functional characterization, analysis, and presentation. SMa performed molecular profiling of the $\mathrm{NGN}_{2}$ iSN. SMi provided technical assistance. MD, LO, and DJA provided resources, reagents, supervision, and edited drafts of the manuscript. AJH, JRM, and RKF-U prepared the manuscript with comments and edits from all authors. All authors contributed to the article and approved the submitted version.

\section{FUNDING}

$\mathrm{AJH}$ was supported by the Australian government research training program scholarship. This study was also supported by funding from a National Health and Medical Research Council of Australia (NHMRC) Program Grant (APP1072113 to DA), Friedreich's Ataxia Research Alliance

\section{REFERENCES}

Abu-Bonsrah, K. D., Viventi, S., Newgreen, D. F., and Dottori, M. (2019). Generation of neural crest progenitors from human pluripotent stem cells. Methods Mol. Biol. 1976, 37-47. doi: 10.1007/978-1-4939-9412-0_3

Alshawaf, A. J., Viventi, S., Qiu, W., D’Abaco, G., Nayagam, B., Erlichster, M., et al. (2018). Phenotypic and functional characterization of peripheral sensory neurons derived from human embryonic stem cells. Sci. Rep. 8:603. doi: 10.1038/s41598-017-19093-0

Axelrod, F. B., and Gold-Von Simson, G. (2007). Hereditary sensory and autonomic neuropathies: types II, III and IV. Orphanet J. Rare Dis. 2:39. doi: 10.1186/1750-1172-2-39

Basch, M. L., Bronner-Fraser, M., and García-Castro, M. I. (2006). Specification of the neural crest occurs during gastrulation and requires Pax7. Nature 441, 218-222. doi: 10.1038/nature04684

Betters, E., Liu, Y., Kjaeldgaard, A., Sundström, E., and García-Castro, M. I. (2010). Analysis of early human neural crest development. Dev. Biol. 344, 578-592. doi: 10.1016/j.ydbio.2010.05.012

Blanchard, J. W., Eade, K. T., Szůcs, A., Lo Sardo, V., Tsunemoto, R. K., Williams, D., et al. (2015). Selective conversion of fibroblasts into peripheral sensory neurons. Nat. Neurosci. 18, 25-35. doi: 10.1038/nn.3887

Boisvert, E. M., Engle, S. J., Hallowell, S. E., Liu, P., Wang, Z.-W., and Li, X.-J. (2015). The specification and maturation of nociceptive neurons from human embryonic stem cells. Sci. Rep. 5:16821. doi: 10.1038/srep16821

Borgland, S. L., Connor, M., and Christie, M. J. (2001). Nociceptin inhibits calcium channel currents in a subpopulation of small nociceptive trigeminal ganglion neurons in mouse. J. Physiol. 536, 35-47. doi: 10.1111/j.1469-7793.2001.t01-100035.x

Castro, J., Harrington, A. M., Garcia-Caraballo, S., Maddern, J., Grundy, L., Zhang, J., et al. (2017). $\alpha$-Conotoxin Vc1.1 inhibits human dorsal root ganglion neuroexcitability and mouse colonic nociception via $\mathrm{GABA}_{\mathrm{B}}$ receptors. Gut 66, 1083-1094. doi: 10.1136/gutjnl-2015-310971

Chambers, S. M., Qi, Y., Mica, Y., Lee, G., Zhang, X.-J., Niu, L., et al. (2012). Combined small-molecule inhibition accelerates developmental timing and converts human pluripotent stem cells into nociceptors. Nat. Biotechnol. 30, 715-720. doi: 10.1038/nbt.2249
USA (to MD), Friedreich Ataxia Research Association Australasia (to MD), Rebecca Cooper Foundation for Medical Research Project Grant (PG2019396 to JRM), and Illawarra Health and Medical Research Institute and University of Wollongong. LO was supported by an NHMRC Boosting Dementia Research Leadership Fellowship (APP1135720).

\section{ACKNOWLEDGMENTS}

MD would like to thank Dr. Ben Rollo for technical advice on lentiviral transduction. RKF-U and JRM thank S. Noosch and Æ. Mittah for insightful discussion and support. Polyethyleneimine was kindly donated by Dr. Diane Ly, IHMRI. א-RIIIJ was a kind gift from B. Olivera, University of Utah.

\section{SUPPLEMENTARY MATERIAL}

The Supplementary Material for this article can be found online at: https://www.frontiersin.org/articles/10.3389/fncel. 2020.600895/full\#supplementary-material.

Chang, W., Berta, T., Kim, Y. H., Lee, S., Lee, S.-Y., and Ji, R.-R. (2018) Expression and role of voltage-gated sodium channels in human dorsal root ganglion neurons with special focus on Nav1.7, species differences and regulation by paclitaxel. Neurosci. Bull. 34, 4-12. doi: 10.1007/s12264-0170132-3

Chaplan, S. R., Guo, H.-Q., Lee, D. H., Luo, L., Liu, C., Kuei, C., et al. (2003). Neuronal hyperpolarization-activated pacemaker channels drive neuropathic pain. J. Neurosci. 23, 1169-1178. doi: 10.1523/ JNEUROSCI.23-04-01169.2003

Chen, S.-R., Cai, Y.-Q., and Pan, H.-L. (2009). Plasticity and emerging role of BKCa channels in nociceptive control in neuropathic pain. J. Neurochem. 110, 352-362. doi: 10.1111/j.1471-4159.2009.06138.x

Cheng, Y.-R., Jiang, B.-Y., and Chen, C.-C. (2018). Acid-sensing ion channels: dual function proteins for chemo-sensing and mechano-sensing. J. Biomed. Sci. 25:46. doi: 10.1186/s12929-018-0448-y

Clark, A. J., Kaller, M. S., Galino, J., Willison, H. J., Rinaldi, S., and Bennett, D. L. H. (2017). Co-cultures with stem cell-derived human sensory neurons reveal regulators of peripheral myelination. Brain 140, 898-913. doi: 10.1093/brain/awx012

Comunanza, V., Carbone, E., Marcantoni, A., Sher, E., and Ursu, D. (2011). Calcium-dependent inhibition of T-type calcium channels by TRPV1 activation in rat sensory neurons. Pflugers Arch. 462, 709-722. doi: 10.1007/s00424-011-1023-5

Cordeiro, S., Finol-Urdaneta, R. K., Köpfer, D., Markushina, A., Song, J., French, R. J., et al. (2019). Conotoxin $\mathrm{\kappa M-RIIIJ,} \mathrm{a} \mathrm{tool} \mathrm{targeting} \mathrm{asymmetric}$ heteromeric Kv1 channels. Proc. Natl. Acad. Sci. U S A 116, 1059-1064. doi: 10.1073/pnas.1813161116

Coward, K., Plumpton, C., Facer, P., Birch, R., Carlstedt, T., Tate, S., et al. (2000). Immunolocalization of SNS/PN3 and NaN/SNS2 sodium channels in human pain states. Pain 85, 41-50. doi: 10.1016/s0304-3959(99)00251-1

Curchoe, C. L., Maurer, J., Mckeown, S. J., Cattarossi, G., Cimadamore, F., Nilbratt, M., et al. (2010). Early acquisition of neural crest competence during hESCs neuralization. PLoS One 5:e13890. doi: 10.1371/journal.pone.0013890

Damasceno, A., França, M. C., and Nucci, A. (2008). Chronic acquired sensory neuron diseases. Eur. J. Neurol. 15, 1400-1405. doi: 10.1111/j.1468-1331.2008. 02332.x 
Davidson, S., Copits, B. A., Zhang, J., Page, G., Ghetti, A., and Gereau, R. W. (2014). Human sensory neurons: membrane properties and sensitization by inflammatory mediators. Pain 155, 1861-1870. doi: 10.1016/j.pain.2014. 06.017

Davis, J. B., Gray, J., Gunthorpe, M. J., Hatcher, J. P., Davey, P. T., Overend, P., et al. (2000). Vanilloid receptor-1 is essential for inflammatory thermal hyperalgesia. Nature 405, 183-187. doi: 10.1038/35012076

Denham, M., Hasegawa, K., Menheniott, T., Rollo, B., Zhang, D., Hough, S., et al. (2015). Multipotent caudal neural progenitors derived from human pluripotent stem cells that give rise to lineages of the central and peripheral nervous system. Stem Cells 33, 1759-1770. doi: 10.1002/stem.1991

Descoeur, J., Pereira, V., Pizzoccaro, A., Francois, A., Ling, B., Maffre, V., et al. (2011). Oxaliplatin-induced cold hypersensitivity is due to remodelling of ion channel expression in nociceptors. EMBO Mol. Med. 3, 266-278. doi: $10.1002 / \mathrm{emmm} .201100134$

Deval, E., Noël, J., Lay, N., Alloui, A., Diochot, S., Friend, V., et al. (2008). ASIC3, a sensor of acidic and primary inflammatory pain. EMBO J. 27, 3047-3055. doi: $10.1038 /$ emboj.2008.213

Eberhardt, E., Havlicek, S., Schmidt, D., Link, A. S., Neacsu, C., Kohl, Z., et al. (2015). Pattern of functional TTX-Resistant dodium channels reveals a developmental stage of human iPSC- and ESC-derived nociceptors. Stem Cell Reports 5, 305-313. doi: 10.1016/j.stemcr.2015.07.010

Finol-Urdaneta, R. K., Belovanovic, A., Micic-Vicovac, M., Kinsella, G. K., McArthur, J. R., and Al-Sabi, A. (2020). Marine toxins targeting Kv1 channels: pharmacological tools and therapeutic scaffolds. Mar. Drugs. 18:173. doi: $10.3390 / \mathrm{md} 18030173$

Fioretti, B., Catacuzzeno, L., Sforna, L., Gerke-Duncan, M. B., van den Maagdenberg, A. M. J. M., Franciolini, F., et al. (2011). Trigeminal ganglion neuron subtype-specific alterations of $\mathrm{Ca}(\mathrm{V}) 2.1$ calcium current and excitability in a Cacnala mouse model of migraine. J. Physiol. 589, 5879-5895. doi: 10.1113/jphysiol.2011.220533

Flegel, C., Schöbel, N., Altmüller, J., Becker, C., Tannapfel, A., Hatt, H., et al. (2015). RNA-Seq analysis of human trigeminal and dorsal root ganglia with a focus on chemoreceptors. PLoS One 10:e0128951. doi: 10.1371/journal.pone. 0128951

François, A., Schüetter, N., Laffray, S., Sanguesa, J., Pizzoccaro, A., Dubel, S., et al. (2015). The low-threshold calcium channel Cav3.2 determines low-threshold mechanoreceptor function. Cell Rep. 10, 370-382. doi: 10.1016/j.celrep.2014. 12.042

Geffeney, S. L., and Goodman, M. B. (2012). How we feel: ion channel partnerships that detect mechanical inputs and give rise to touch and pain perception. Neuron 74, 609-619. doi: 10.1016/j.neuron.2012.04.023

Giacobassi, M. J., Leavitt, L. S., Raghuraman, S., Alluri, R., Chase, K., FinolUrdaneta, R. K., et al. (2020). An integrative approach to the facile functional classification of dorsal root ganglion neuronal subclasses. Proc. Natl. Acad. Sci. U S A 117, 5494-5501. doi: 10.1073/pnas.1911382117

González, A., Herrera, G., Ugarte, G., Restrepo, C., Piña, R., Pertusa, M., et al. (2017). IKD Current in cold transduction and damage-triggered cold hypersensitivity. Adv. Exp. Med. Biol. 1015, 265-277. doi: 10.1007/978-3-31962817-2_14

Groysman, M., Shoval, I., and Kalcheim, C. (2008). A negative modulatory role for rho and rho-associated kinase signaling in delamination of neural crest cells. Neural Dev. 3:27. doi: 10.1186/1749-8104-3-27

Gu, Q., and Lee, L.-Y. (2010). Acid-sensing ion channels and pain. Pharmaceuticals 3, 1411-1425. doi: 10.3390/ph3051411

Gutman, G. A., Chandy, K. G., Grissmer, S., Lazdunski, M., Mckinnon, D., Pardo, L. A., et al. (2005). International union of pharmacology. LIII. Nomenclature and molecular relationships of voltage-gated potassium channels. Pharmacol. Rev. 57, 473-508. doi: 10.1124/pr.57.4.10

Haberberger, R. V., Barry, C., Dominguez, N., and Matusica, D. (2019). Human dorsal root ganglia. Front. Cell. Neurosci. 13:271. doi: 10.3389/fncel.2019.00271

Han, C., Estacion, M., Huang, J., Vasylyev, D., Zhao, P., Dib-Hajj, S. D., et al. (2015). Human Nav1.8: enhanced persistent and ramp currents contribute to distinct firing properties of human DRG neurons. J. Neurophysiol. 113, 3172-3185. doi: 10.1152/jn.00113.2015

Ho, C., and O'Leary, M. E. (2011). Single-cell analysis of sodium channel expression in dorsal root ganglion neurons. Mol. Cell. Neurosci. 46, 159-166. doi: $10.1016 /$ j.mcn.2010.08.017
Hotta, R., Pepdjonovic, L., Anderson, R. B., Zhang, D., Bergner, A. J., Leung, J., et al. (2009). Small-molecule induction of neural crest-like cells derived from human neural progenitors. Stem Cells 27, 2896-2905. doi: 10.1002/ stem. 208

Johnson, P. C., Doll, S. C., and Cromey, D. W. (1986). Pathogenesis of diabetic neuropathy. Ann. Neurol. 19, 450-457. doi: 10.1002/ana.4101 90505

Kaczmarek, L. K., and Zhang, Y. (2017). Kv3 channels: enablers of rapid firing, neurotransmitter release and neuronal endurance. Physiol. Rev. 97, 1431-1468. doi: 10.1152/physrev.00002.2017

Keenan, B., Finol-Urdaneta, R. K., Hope, A., Bremner, J. B., Kavallaris, M., LucenaAgell, D., et al. (2020). $N$-alkylisatin-based microtubule destabilizers bind to the colchicine site on tubulin and retain efficacy in drug resistant acute lymphoblastic leukemia cell lines with less in vitro neurotoxicity. Cancer Cell Int. 20:170. doi: 10.1186/s12935-020-01251-6

Kim, K., Ossipova, O., and Sokol, S. Y. (2015). Neural crest specification by inhibition of the ROCK/myosin II pathway. Stem Cells 33, 674-685. doi: 10.1002/stem.1877

Kouranova, E. V., Strassle, B. W., Ring, R. H., Bowlby, M. R., and Vasilyev, D. V. (2008). Hyperpolarization-activated cyclic nucleotide-gated channel mRNA and protein expression in large versus small diameter dorsal root ganglion neurons: correlation with hyperpolarization-activated current gating. Neuroscience 153, 1008-1019. doi: 10.1016/j.neuroscience.2008. 03.032

Kuntzer, T., Antoine, J.-C., and Steck, A. J. (2004). Clinical features and pathophysiological basis of sensory neuronopathies (ganglionopathies). Muscle Nerve 30, 255-268. doi: 10.1002/mus.20100

Leo, M., Schmitt, L.-I., Erkel, M., Melnikova, M., Thomale, J., and Hagenacker, T. (2017). Cisplatin-induced neuropathic pain is mediated by upregulation of $\mathrm{N}$-type voltage-gated calcium channels in dorsal root ganglion neurons. Exp. Neurol. 288, 62-74. doi: 10.1016/j.expneurol.2016.11.003

Lin, S.-H., Cheng, Y.-R., Banks, R. W., Min, M.-Y., Bewick, G. S., and Chen, C.-C. (2016). Evidence for the involvement of ASIC3 in sensory mechanotransduction in proprioceptors. Nat. Commun. 7:11460. doi: $10.1038 /$ ncomms 11460

Liu, P. W., and Bean, B. P. (2014). Kv2 channel regulation of action potential repolarization and firing patterns in superior cervical ganglion neurons and hippocampal CA1 pyramidal neurons. J. Neurosci. 34, 4991-5002. doi: 10.1523/JNEUROSCI.1925-13.2014

Luna-Ramirez, K., Csoti, A., McArthur, J. R., Chin, Y. K. Y., Anangi, R., Del Carmen Najera, R., et al. (2020). Structural basis of the potency and selectivity of Urotoxin, a potent Kv1 blocker from scorpion venom. Biochem. Pharmacol. 174:113782. doi: 10.1016/j.bcp.2019.113782

Maczkowiak, F., Matéos, S., Wang, E., Roche, D., Harland, R., and MonsoroBurq, A. H. (2010). The Pax3 and Pax7 paralogs cooperate in neural and neural crest patterning using distinct molecular mechanisms, in Xenopus laevis embryos. Dev. Biol. 340, 381-396. doi: 10.1016/j.ydbio.2010. 01.022

Martinez, A. R. M., Nunes, M. B., Nucci, A., and França, M. C. (2012). Sensory neuronopathy and autoimmune diseases. Autoimmune Dis. 2012:873587. doi: $10.1155 / 2012 / 873587$

Mayer, M. L., and Westbrook, G. L. (1983). A voltage-clamp analysis of inward (anomalous) rectification in mouse spinal sensory ganglion neurones. J. Physiol. 340, 19-45. doi: 10.1113/jphysiol.1983.sp014747

McArthur, J. R., Finol-Urdaneta, R. K., and Adams, D. J. (2019). Analgesic transient receptor potential vanilloid-1-active compounds inhibit native and recombinant T-type calcium channels. Br. J. Pharmacol. 176, 2264-2278. doi: 10.1111/bph.14676

McDermott, L. A., Weir, G. A., Themistocleous, A. C., Segerdahl, A. R., Blesneac, I., Baskozos, G., et al. (2019). Defining the functional role of $\mathrm{Na}_{V} 1.7$ in human nociception. Neuron 101, 905.e8-919.e8. doi: 10.1016/j.neuron.2019. 01.047

McKeown, S. J., Lee, V. M., Bronner-Fraser, M., Newgreen, D. F., and Farlie, P. G. (2005). Sox10 overexpression induces neural crest-like cells from all dorsoventral levels of the neural tube but inhibits differentiation. Dev. Dyn. 233, 430-444. doi: 10.1002/dvdy.20341

Meents, J. E., Bressan, E., Sontag, S., Foerster, A., Hautvast, P., Rösseler, C., et al. (2019). The role of Nav1.7 in human nociceptors: insights from human induced 
pluripotent stem cell-derived sensory neurons of erythromelalgia patients. Pain 160, 1327-1341. doi: 10.1097/j.pain.0000000000001511

Milescu, L. S., Bean, B. P., and Smith, J. C. (2010). Isolation of somatic $\mathrm{Na}^{+}$ currents by selective inactivation of axonal channels with a voltage prepulse. J. Neurosci. 30, 7740-7748. doi: 10.1523/JNEUROSCI.6136-09.2010

Murdoch, B., DelConte, C., and García-Castro, M. I. (2012). Pax7 lineage contributions to the mammalian neural crest. PLoS One 7:e41089. doi: 10.1371/journal.pone.0041089

Na-Phuket, T. R., and Covarrubias, M. (2009). Kv4 channels underlie the subthreshold-operating A-type K-current in nociceptive dorsal root ganglion neurons. Front. Mol. Neurosci. 2:3. doi: 10.3389/neuro.02.003.2009

Nickolls, A. R., Lee, M. M., Espinoza, D. F., Szczot, M., Lam, R. M., Wang, Q., et al. (2020). Transcriptional programming of human mechanosensory neuron subtypes from pluripotent stem cells. Cell Rep. 30, 932.e7-946.e7. doi: 10.1016/j. celrep.2019.12.062

Omerbašić, D., Schuhmacher, L.-N., Bernal Sierra, Y.-A., St John Smith, E., and Lewin, G. R. (2015). ASICs and mammalian mechanoreceptor function. Neuropharmacology 94, 80-86. doi: 10.1016/j.neuropharm.2014.12.007

Page, A. J., Brierley, S. M., Martin, C. M., Price, M. P., Symonds, E., Butler, R., et al. (2005). Different contributions of ASIC channels 1a, 2 and 3 in gastrointestinal mechanosensory function. Gut 54, 1408-1415. doi: 10.1136/gut.2005.071084

Papalampropoulou-Tsiridou, M., Labrecque, S., Godin, A. G., De Koninck, Y., and Wang, F. (2020). Differential expression of acid-sensing ion channels in mouse primary afferents in naïve and injured conditions. Front. Cell. Neurosci. 14:103. doi: $10.3389 /$ fncel.2020.00103

Park, J., and Luo, Z. D. (2010). Calcium channel functions in pain processing. Channels 4, 510-517. doi: 10.4161/chan.4.6.12869

Pathak, D., Guan, D., and Foehring, R. C. (2016). Roles of specific Kv channel types in repolarization of the action potential in genetically identified subclasses of pyramidal neurons in mouse neocortex. J. Neurophysiol. 115, 2317-2329. doi: $10.1152 /$ jn. 01028.2015

Pearce, R. J., and Duchen, M. R. (1994). Differential expression of membrane currents in dissociated mouse primary sensory neurons. Neuroscience 63, 1041-1056. doi: 10.1016/0306-4522(94)90571-1

Pitake, S., Middleton, L. J., Abdus-Saboor, I., and Mishra, S. K. (2019). Inflammation induced sensory nerve growth and pain hypersensitivity requires the N-type calcium channel Cav2.2. Front. Neurosci. 13:1009. doi: 10.3389/fnins.2019.01009

Poolos, N. P. (2004). The yin and yang of the H-channel and its role in epilepsy. Epilepsy Curr. 4, 3-6. doi: 10.1111/j.1535-7597.2004.04101.x

Price, M. P., Lewin, G. R., McIlwrath, S. L., Cheng, C., Xie, J., Heppenstall, P. A., et al. (2000). The mammalian sodium channel BNC1 is required for normal touch sensation. Nature 407, 1007-1011. doi: 10.1038/35039512

Rasband, M. N., Park, E. W., Vanderah, T. W., Lai, J., Porreca, F., and Trimmer, J. S. (2001). Distinct potassium channels on pain-sensing neurons. Proc. Natl. Acad. Sci. U S A 98, 13373-13378. doi: 10.1073/pnas.231376298

Ritter, A. M., and Mendell, L. M. (1992). Somal membrane properties of physiologically identified sensory neurons in the rat: effects of nerve growth factor. J. Neurophysiol. 68, 2033-2041. doi: 10.1152/jn.1992.68.6.2033

Robinson, R. B., and Siegelbaum, S. A. (2003). Hyperpolarization-activated cation currents: from molecules to physiological function. Annu. Rev. Physiol. 65, 453-480. doi: 10.1146/annurev.physiol.65.092101. 142734

Rostock, C., Schrenk-Siemens, K., Pohle, J., and Siemens, J. (2018). Human vs. mouse nociceptors-similarities and differences. Neuroscience 387, 13-27. doi: 10.1016/j.neuroscience.2017.11.047

Rutlin, M., Ho, C.-Y., Abraira, V. E., Cassidy, C., Woodbury, C. J., and Ginty, D. D. (2014). The cellular and molecular basis of direction selectivity of A $\delta$-LTMRs. Cell 159, 1640-1651. doi: 10.1016/j.cell.2014.11.038

Sartiani, L., Mannaioni, G., Masi, A., Romanelli, M. N., and Cerbai, E. (2017). The hyperpolarization-activated cyclic nucleotide-gated channels: from biophysics to pharmacology of a unique family of ion channels. Pharmacol. Rev. 69, 354-395. doi: 10.1124/pr.117.014035

Schoepf, C. L., Zeidler, M., Spiecker, L., Kern, G., Lechner, J., Kummer, K. K., et al. (2020). Selected ionotropic receptors and voltage-gated ion channels: more functional competence for human induced pluripotent stem cell (iPSC)derived nociceptors. Brain Sci. 10:344. doi: 10.3390/brainsci10060344
Schrenk-Siemens, K., Wende, H., Prato, V., Song, K., Rostock, C., Loewer, A., et al. (2015). PIEZO2 is required for mechanotransduction in human stem cell-derived touch receptors. Nat. Neurosci. 18, 10-16. doi: 10.1038/nn.3894

Schwaid, A.G., Krasowka-Zoladek, A., Chi, A., and Cornella-Taracido, I. (2018). Comparison of the rat and human dorsal root ganglion proteome. Sci. Rep. 8:13469. doi: 10.1038/s41598-018-31189-9

Sghirlanzoni, A., Pareyson, D., and Lauria, G. (2005). Sensory neuron diseases. Lancet Neurol. 4, 349-361. doi: 10.1016/S1474-4422(05) 70096-X

Shields, S. D., Ahn, H.-S., Yang, Y., Han, C., Seal, R. P., Wood, J. N., et al. (2012). Nav1.8 expression is not restricted to nociceptors in mouse peripheral nervous system. Pain 153, 2017-2030. doi: 10.1016/ j.pain.2012.04.022

Shiers, S., Klein, R. M., and Price, T. J. (2020). Quantitative differences in neuronal subpopulations between mouse and human dorsal root ganglia demonstrated with RNAscope in situ hybridization. Pain 161, 2410-2424. doi: 10.1097/j.pain. 0000000000001973

Stieber, J., Stöckl, G., Herrmann, S., Hassfurth, B., and Hofmann, F. (2005). Functional expression of the human HCN3 channel. J. Biol. Chem. 280, 34635-34643. doi: 10.1074/jbc.M502508200

Usoskin, D., Furlan, A., Islam, S., Abdo, H., Lönnerberg, P., Lou, D., et al. (2015). Unbiased classification of sensory neuron types by large-scale single-cell RNA sequencing. Nat. Neurosci. 18, 145-153. doi: 10.1038/nn.3881

Vacher, H., Alami, M., Crest, M., Possani, L. D., Bougis, P. E., and MartinEauclaire, M. F. (2002). Expanding the scorpion toxin $\alpha$-KTX 15 family with AmmTX3 from androctonus mauretanicus. Eur. J. Biochem. 269, 6037-6041. doi: 10.1046/j.1432-1033.2002.03294.x

Wainger, B. J., Buttermore, E. D., Oliveira, J. T., Mellin, C., Lee, S., Saber, W. A., et al. (2015). Modeling pain in vitro using nociceptor neurons reprogrammed from fibroblasts. Nat. Neurosci. 18, 17-24. doi: 10.1038/ nn.3886

Walker, K. M., Urban, L., Medhurst, S. J., Patel, S., Panesar, M., Fox, A. J., et al. (2003). The VR1 antagonist capsazepine reverses mechanical hyperalgesia in models of inflammatory and neuropathic pain. J. Pharmacol. Exp. Ther. 304, 56-62. doi: 10.1124/jpet.102.042010

Young, G. T., Gutteridge, A., Fox, H. D. E., Wilbrey, A. L., Cao, L., Cho, L. T., et al. (2014). Characterizing human stem cell-derived sensory neurons at the single-cell level reveals their ion channel expression and utility in pain research. Mol. Ther. 22, 1530-1543. doi: 10.1038/mt.2014.86

Zhang, H., and Dougherty, P. M. (2014). Enhanced excitability of primary sensory neurons and altered gene expression of neuronal ion channels in dorsal root ganglion in paclitaxel-induced peripheral neuropathy. Anesthesiology 120 , 1463-1475. doi: 10.1097/ALN.0000000000000176

Zhang, X., Priest, B. T., Belfer, I., and Gold, M.S. (2017). Voltage-gated $\mathrm{Na}^{+}$currents in human dorsal root ganglion neurons. eLife 6:e23235. doi: $10.7554 /$ eLife.23235

Zheng, Y., Liu, P., Bai, L., Trimmer, J. S., Bean, B. P., and Ginty, D. D. (2019). Deep sequencing of somatosensory neurons reveals molecular determinants of intrinsic physiological properties. Neuron 103, 598.e7-616.e7. doi: 10.1016/j. neuron.2019.05.039

Zylka, M. J., Dong, X., Southwell, A. L., and Anderson, D. J. (2003). Atypical expansion in mice of the sensory neuron-specific Mrg G proteincoupled receptor family. Proc. Natl. Acad. Sci. U S A 100, 10043-10048. doi: 10.1073/pnas.1732949100

Conflict of Interest: The authors declare that the research was conducted in the absence of any commercial or financial relationships that could be construed as a potential conflict of interest.

Copyright (c) 2020 Hulme, McArthur, Maksour, Miellet, Ooi, Adams, FinolUrdaneta and Dottori. This is an open-access article distributed under the terms of the Creative Commons Attribution License (CC BY). The use, distribution or reproduction in other forums is permitted, provided the original author(s) and the copyright owner(s) are credited and that the original publication in this journal is cited, in accordance with accepted academic practice. No use, distribution or reproduction is permitted which does not comply with these terms. 